\title{
6 Mediennutzung und Fettleibigkeit
}

Ein letzter Themenschwerpunkt des Berliner Längsschnitts Medien war die Untersuchung des Zusammenhangs zwischen häuslicher Mediennutzung und der körperlichen Entwicklung der Schülerinnen und Schüler. Hier sollte vor allem der Frage nach der Entstehung von Fettleibigkeit bzw. Übergewicht durch exzessiven Medienkonsum nachgegangen werden.

Zunächst wird wiederum der allgemeine Forschungsstand zum Thema Mediennutzung und Fettleibigkeit zusammengefasst (Kapitel 6.1), dann die Ergebnisse des Berliner Längsschnitts Medien präsentiert (Kapitel 6.2) und diese schließlich kritisch vor dem Hintergrund der präsentierten Forschungslage diskutiert (Kapitel 6.3).

\subsection{Theoretische Grundlagen und Literaturüberblick - Mediennutzung und Fettleibigkeit}

Theoretische Annahmen und wissenschaftliche Untersuchungen zum Zusammenhang bestimmter Formen der Mediennutzung, hier insbesondere einer zeitlich ausgedehnten Nutzung, und Übergewicht bzw. Fettleibigkeit, finden ihren Ursprung zunächst in einer allgemeinen Zunahme übergewichtiger bzw. adipöser Kinder, Jugendlicher und Erwachsener in den letzten Jahrzehnten. So berichtet die Kaiser Family Foundation für die USA: „Since 1980 the proportion of overweight children ages 6-11 has more than doubled, and the rate for adolescents has tripled. Today about $10 \%$ of 2 -to 5 -year-olds and $15 \%$ of 6 - to 19 -year-olds are overweight. Taking into consideration the proportion who are "at risk" of being overweight, the current percentages double to $20 \%$ for children of ages 2-5 and $30 \%$ for kids ages 6-19" (Kaiser Family Foundation, 2004, S.1). Auch in der europäischen Region hat sich laut der Weltgesundheitsorganisation (WHO) die Prävalenz der Adipositas in den letzten beiden Jahrzenten verdreifacht und es wurde im letzten Bericht aus dem Jahr 2007 für 2010 bei einer gleichbleibenden Entwicklung eine Anzahl von 15 Millionen adipösen Kindern und Jugendlichen geschätzt (Branca, Nikogosian \& Lobstein, 2007). Der Kinder- und Jugendgesundheitssurvey (KiGGS) berichtet schließlich für Deutschland einen Anteil von 15 Prozent bei 3- bis 17-jährigen Kindern und Jugendlichen, die als übergewichtig (inklusive adipös) klassifiziert werden und einen Anteil von 6 Prozent, welche als adipös eingestuft werden (Kurth, B. \& Schaffrath, 2007), wobei in beiden 
Kategorien eine Zunahme mit dem Alter festzustellen ist; übergewichtig: 3-6jährige Kinder - 9\%, 7-10-jährige Kinder - 15\%, 11-13-jährige Kinder - 19\%, 14-17-jährige Kinder - 17\%; adipös: 3-6-jährige Kinder - 2.9\%, 7-10-jährige Kinder - 6.4\%, 11-13-jährige Kinder - 7.2\%; 14-17-jährige Kinder - 8.5\%.

Darüber hinaus zeigen epidemiologische Untersuchungen im Allgemeinen eine zunehmende Bewegungsarmut bei Kindern und Jugendlichen sowie eine allgemeine Abnahme der körperlichen Fitness. So wird im ersten deutschen Kinder- und Jugendsportbericht ausgeführt, dass im Jahre 2000 gegenüber 1975 eine durchschnittliche physiologische Leistungsabnahme von 10 Prozent feststellbar ist, mit den größten Unterschieden im Bereich der Grundlagenausdauer und Beweglichkeit (Schmidt, W., Hartmann-Tews \& Brettschneider, 2003; ein Überblick der internationalen Studienlage bei Bös, 2003).

„During the same period in which childhood obesity has increased so dramatically, there has also been an explosion in media targeted to children: TV shows and videos, specialized cable networks, video games, computer activities and Internet web sites. Children today spend $[\ldots]$ the equivalent of a full time job [using media]" (Kaiser Family Foundation, 2004, S.1). Aus diesem Grund wurde die Thematik Übergewicht und Fettleibigkeit oft auch in der Trias Fettleibigkeit, Bewegungsmangel, Medienkonsum betrachtet, wobei Letzterem eine wichtige Erklärungskraft für beide oben beschriebenen Entwicklungen zugesprochen wurde.

Welchen Stellenwert dabei die Nutzung elektronischer Medien im komplexen Zusammenspiel biopsychosozialer Schutz- und Risikofaktoren einnimmt, wurde u. a. von Monasta und Kollegen (Monasta et al., 2010) in ihrem Review systematischer Reviews (wie sie es selbst bezeichnen) versucht zu veranschaulichen (vgl. Abbildung 78). So kommen Monasta und Kollegen (2010) insbesondere in Bezug auf die frühkindlichen Einflussfaktoren zu folgendem Schluss:

"The results $[\ldots]$ indicate that breastfeeding may be a protective factor for later overweight and obesity, while obesity in infancy, rapid infant growth, maternal diabetes, maternal smoking, short sleep duration, less than $30 \mathrm{~min}$ of daily physical activity and consumption of sugar-sweetened beverages may be considered as risk factors. Parental obesity, restricted or excessive intrauterine growth, small or large size at birth, television viewing, food insecurity and low socioeconomic status can also be included among risk factors, although it is difficult to estimate the size of their effect. Among all these possible determinants, maternal smoking, breastfeeding, infant size and growth, short sleep duration and television viewing, including video and computer games, are those supported by better-quality systematic reviews." (Monasta et al., 2010, S. 703) 


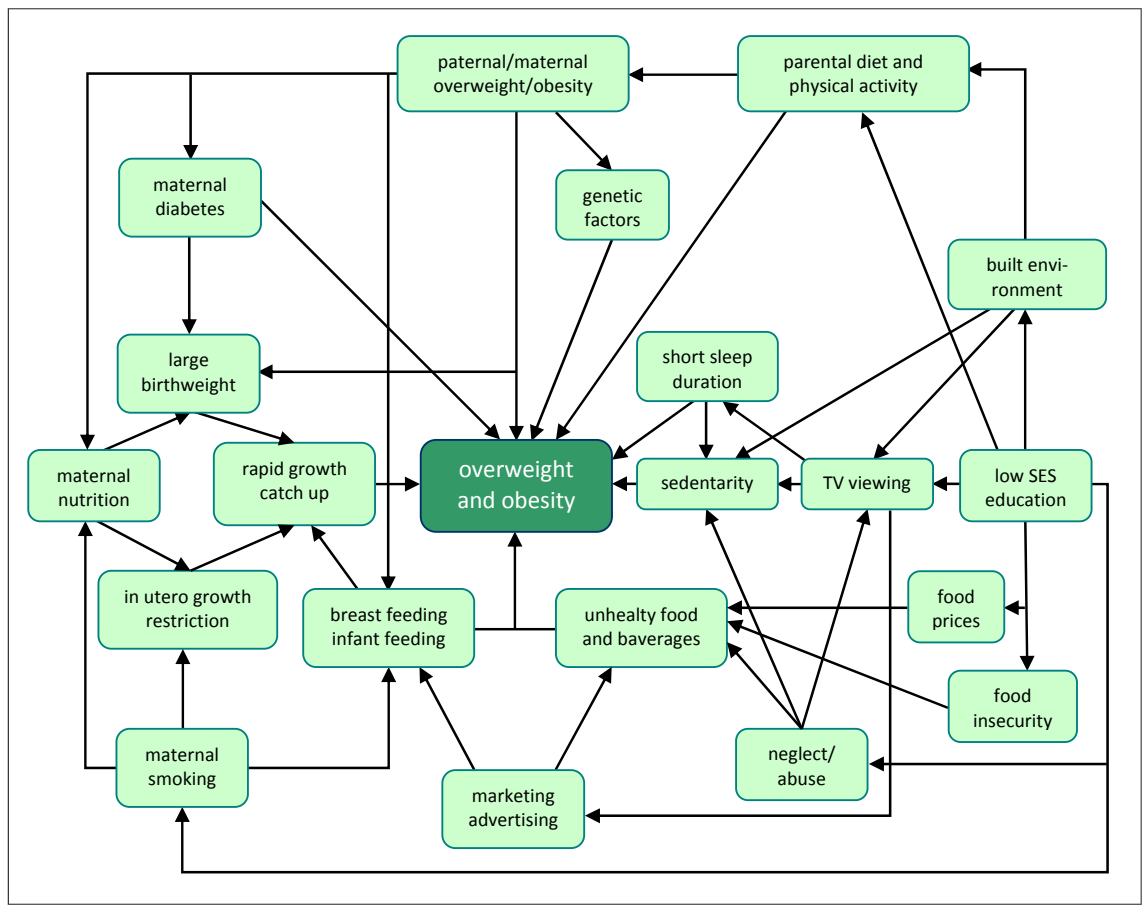

Abbildung 78. Erklärungsmodell Übergewicht und Adipositas aus Monasta und Kollegen (Monasta et al., 2010).

\subsubsection{Forschungsergebnisse zum Zusammenhang Mediennutzung und Fettleibigkeit}

Analog zu Kapitel 3 (Mediennutzung und Schulleistung) und Kapitel 4 (Gewaltmediennutzung und Gewaltverhalten) wurde auch für den Zusammenhang zwischen Variablen der Mediennutzung und Fettleibigkeit/Übergewicht/Adipositas eine Literaturrecherche einschlägiger Studien ${ }^{203}$ der Jahre 2001 bis 2011 durchgeführt, wobei wiederum nur Studien in die Übersicht mit aufgenommen wurden, die zumindest eine kindliche oder jugendliche Substichprobe beinhalteten.

203 Für den Zeitraum 2001 bis 2011 wurden die Datenbanken PsychInfo, Pubmed und google scholar mit den Suchbegriffen Mediennutzung, Fernseh, TV, Videospiel, Computerspiel, media use, television, tv, video games, computer games, jeweils in Kombination (UND / AND) mit Fettleibigkeit, Übergewicht, Adipositas, obesity, body fatness, adiposity durchsucht. Als Treffer galten Experimente, Querschnitts- und Längsschnittuntersuchungen, an denen Kinder und Jugendliche teilgenommen haben, sowie Reviews oder Metaanalysen, die sich ebenfalls mit dieser Population befassten. 
Es wurden insgesamt 120 Studien bzw. Artikel identifiziert, welche den gesetzten Kriterien entsprachen: eine Metaanalyse (Marshall et al., 2004), 20 Längsschnittstudien (Berkey, Rockett, Gillman \& Colditz, 2003; Proctor et al., 2003; Viner \& Cole, 2005; Davison, Marshall \& Birch, 2006; Hancox \& Poulton, 2006; Blair et al., 2007; Delmas et al., 2007; Gable, Chang \& Krull, 2007; Henderson, 2007; Hesketh, Wake, Graham \& Waters, 2007; Must et al., 2007; Danner, 2008; Dubois, Farmer, Girard \& Peterson, 2008; Landhuis, Poulton, Welch \& Hancox, 2008; Laurson, Eisenmann \& Moore, 2008; Parsons, Manor \& Power, 2008; MacFarlane, Cleland, Crawford, Campbell \& Timperio, 2009; Barnett et al., 2010; Mota, Ribeiro, Carvalho, Santos \& Martins, 2010; Zimmerman \& Bell, 2010), 76 Querschnittsstudien (Crespo et al., 2001; Janz et al., 2002; Lowry, 2002; Arluk, Branch, Swain \& Dowling, 2003; Giammattei, Blix, Marshak, Wollitzer \& Pettitt, 2003; Storey, Forshee, Weaver \& Sansalone, 2003; Tremblay \& Willms, 2003; Wake, Hesketh \& Waters, 2003; Waller, Du \& Popkin, 2003; Janssen, Katzmarzyk, Boyce, King \& Pickett, 2004; Stettler, Signer \& Suter, 2004; Vandewater, Shim \& Caplovitz, 2004; Kautiainen, Koivusilta, Lintonen, Virtanen \& Rimpela, 2005; Burke et al., 2006; Carvalhal, Padez, Moreira \& Rosado, 2006; Ekelund et al., 2006; Heelan \& Eisenmann, 2006; Utter, Scragg \& Schaaf, 2006; Vandewater \& Huang, 2006; Adachi-Mejia et al., 2007; Gomez et al., 2007; Lajunen et al., 2007; Laxmaiah, Nagalla, Vijayaraghavan \& Nair, 2007; van Zutphen, Bell, Kremer \& Swinburn, 2007; Kuriyan, Bhat, Thomas, Vaz \& Kurpad, 2007; Mendoza, Zimmerman \& Christakis, 2007; Schneider, M., Dunton \& Cooper, 2007; Wang, Y. et al., 2007; Beyerlein, Fahrmeir, Mansmann \& Toschke, 2008; Eisenmann, Bartee, Smith, Welk \& Fu, 2008; Lagiou \& Parava, 2008; Laurson, Eisenmann, Welk, et al., 2008; Singh, Kogan, Van Dyck \& Siahpush, 2008; Toschke, von Kries, Beyerlein \& Ruckinger, 2008; VicenteRodriguez et al., 2008; Wells et al., 2008; Xu, Li, Ware \& Owen, 2008; Aucote \& Cooper, 2009; Chang \& Nayga, 2009; Fulton et al., 2009; Khader et al., 2009; Jackson, D. M., Djafarian, Stewart \& Speakman, 2009; Lajous et al., 2009; McDonald, Baylin, Arsenault, Mora-Plazas \& Villamor, 2009; Mihas et al., 2009; Montgomery-Reagan, Bianco, Heh, Rettos \& Huston, 2009; Morales-Ruan, Hernandez-Prado, Gomez-Acosta, Shamah-Levy \& Cuevas-Nasu, 2009; Nagel et al., 2009; Ramic et al., 2009; Russ, Larson, Franke \& Halfon, 2009; Steffen, Dai, Fulton \& Labarthe, 2009; Sun, Sekine \& Kagamimori, 2009; Anderson, S. E. \& Whitaker, 2010; Bishwalata, Singh, Singh, Devi \& Singh, 2010; de Gouw et al., 2010; del Mar Bibiloni et al., 2010; Garcinuno, Lopez, Alonso \& Garcia, 2010; Hardy, Denney-Wilson, Thrift, Okely \& Baur, 2010; Lazarou \& Soteriades, 2010; Li, X. et al., 2010; Liou, Liou \& Chang, 2010; Munakata et al., 2010; Oliver, Schluter, Rush, Schofield \& Paterson, 2010; Papandreou, Malindretos \& Rousso, 2010; Rivera, Silva, Silva, Oliveira \& Carvalho, 2010; Rosen- 
kranz, Bauer \& Dzewaltowski, 2010; Sasaki et al., 2010; Tang et al., 2010; Wen, Merom, Rissel \& Simpson, 2010; Yen, C. F. et al., 2010; Bener et al., 2011; Danielsen et al., 2011; Lehto, Ray, Lahti-Koski \& Roos, 2011; Ozmert, Ozdemir, Pektas, Uckardes \& Yurdakok, 2011; Perez et al., 2011; Plachta-Danielzik, Landsberg, Lange, Langnäse \& Müller, 2011) sowie 8 Interventionsstudien (Robinson, 2001; Epstein et al., 2008; Mhurchu et al., 2008; Plachta-Danielzik, Kriwy \& Müller, 2008; Salmon, Ball, Hume, Booth \& Crawford, 2008; Robinson et al., 2010; Epstein, Roemmich, Cavanaugh \& Paluch, 2011; Taveras et al., 2011). Darüber hinaus wurden in der durchgeführten Literaturanalyse weitere 15 Reviews und Überblicksartikel identifiziert, in denen Befunde anderer Studien zusammengefasst werden (Clocksin, Watson \& Ransdell, 2002; Caroli, Argentieri, Cardone \& Masi, 2004; Kaiser Family Foundation, 2004; Brettschneider, Naul, Bünemann \& Hoffmann, 2006; Bryant, Lucove, Evenson \& Marshall, 2007; Jordan, 2007; Maibach, 2007; Stephenson \& Banet-Weiser, 2007; Dennison \& Edmunds, 2008; Jordan \& Robinson, 2008; Rey-López, Vicente-Rodríguez, Biosca \& Moreno, 2008; Hebebrand \& Hinney, 2009; Must \& Parisi, 2009; Brandt, Moß, Berg \& Wabitsch, 2010; Monasta et al., 2010). Für die Längsschnittstudien können die genauen Details der Studien (Stichprobe, abhängige und unabhängige Variablen, Auswertungsmethodik - soweit berichtet - und Befunde) Tabelle 76 entnommen werden. Aufgrund der Vielzahl der insgesamt aufgefundenen Studien wurden Reviews und Überblicksartikel sowie Querschnittsstudien und Interventionsstudien zwar für einen zusammenfassenden Überblick berücksichtigt, nicht jedoch in separaten Tabellen aufgeführt. Die Metaanalyse wird ausführlich im Text dargestellt.

Zusammengefasst liefern die genannten nationalen wie internationalen Studien zahlreiche Belege für einen Zusammenhang zwischen einer intensiven Mediennutzung bzw. -ausstattung und einem erhöhten Risiko für Übergewicht und Fettleibigkeit: Ausstattung mit Mediengeräten (Adachi-Mejia et al., 2007; Delmas et al., 2007), Fernsehkonsum (Proctor et al., 2003; Viner \& Cole, 2005; Davison et al., 2006; Hancox \& Poulton, 2006; Delmas et al., 2007; Gable et al., 2007; Henderson, 2007; Danner, 2008; Dubois et al., 2008; Landhuis et al., 2008; Parsons et al., 2008; Smith, Rhodes, Naylor \& McKay, 2008; MacFarlane et al., 2009; Zimmerman \& Bell, 2010; Crespo et al., 2001; Janz et al., 2002; Lowry, 2002; Giammattei et al., 2003; Storey et al., 2003; Ekelund et al., 2006; Heelan \& Eisenmann, 2006; Utter et al., 2006; Gomez et al., 2007; Laxmaiah et al., 2007; van Zutphen et al., 2007; Kuriyan et al., 2007; Beyerlein et al., 2008; Eisenmann et al., 2008; Singh et al., 2008; Toschke et al., 2008; Vicente-Rodriguez et al., 2008; Wells et al., 2008; Xu et al., 2008; Chang \& Nayga, 2009; Khader et al., 2009; Jackson, D. M. et al., 2009; Montgomery-Reagan et al., 2009; Nagel et al., 2009; Steffen et al., 2009; Anderson, S. E. \& Whitaker, 2010; 
de Gouw et al., 2010; Garcinuno et al., 2010; Li, X. et al., 2010; Liou et al., 2010; Oliver et al., 2010; Papandreou et al., 2010; Rivera et al., 2010; Rosenkranz et al., 2010; Tang et al., 2010; Lehto et al., 2011; Perez et al., 2011; Caroli et al., 2004; Bryant et al., 2007; Jordan, 2007; Dennison \& Edmunds, 2008; Jordan \& Robinson, 2008; Hebebrand \& Hinney, 2009; Brandt et al., 2010; Monasta et al., 2010), Fernseh- und Computerspielkonsum (Marshall et al., 2004; Tremblay \& Willms, 2003; Stettler et al., 2004; Kautiainen et al., 2005; Laurson, Eisenmann, Welk, et al., 2008), Fernseh- und PC-Nutzung (Fernsehkonsum und PC-Nutzung: Tremblay \& Willms, 2003; Stettler et al., 2004; Kautiainen et al., 2005), Mediennutzung im Allgemeinen (Berkey et al., 2003; Blair et al., 2007; Hesketh et al., 2007; Epstein et al., 2011; Lajunen et al., 2007; Lagiou \& Parava, 2008; Morales-Ruan et al., 2009; Russ et al., 2009; Wen et al., 2010; Danielsen et al., 2011; Plachta-Danielzik et al., 2011; Clocksin et al., 2002; Kaiser Family Foundation, 2004; Brettschneider et al., 2006; Maibach, 2007; Stephenson \& Banet-Weiser, 2007; Rey-López et al., 2008; Must \& Parisi, 2009).

Teilweise berichten Studien aber auch gemischte Ergebnisse. So begrenzen Lazarazou und Soteriades (2010) den gefunden Zusammenhang zwischen Fernsehkonsum und Übergewicht auf Mädchen. Munakata und Kollegen (2010) tun dies auf Jungen (ähnliches berichten Barnett und Kollegen für Medienzeiten insgesamt; Barnett et al., 2010). Sun und Kollegen (Sun et al., 2009) finden zwar einen Zusammenhang zwischen Fernsehkonsum bzw. Computerspielen und Übergewicht, beschränken Letzteren allerdings auf die Mädchen. Weitere Studien berichten bei gleichzeitiger Betrachtung von Fernseh- und Computerspielkonsum bzw. PC-Nutzung lediglich einen Zusammenhang zwischen Fernsehnutzungszeiten und Übergewicht (Wake et al., 2003; Janssen et al., 2004; Burke et al., 2006; Mendoza et al., 2007; Aucote \& Cooper, 2009; Bishwalata et al., 2010; del Mar Bibiloni et al., 2010). Dem gegenüber stehen wiederum Studien, die dies genau in umgekehrter Weise tun (Vandewater et al., 2004; Carvalhal et al., 2006; Schneider, M. et al., 2007).

Deutlich seltener wird explizit über einen fehlenden Zusammenhang zwischen der Nutzung digitaler Bildschirmmedien und Übergewicht, Fettleibigkeit oder Adipositas berichtet: Fernsehkonsum (Vandewater \& Huang, 2006; McDonald et al., 2009; Sasaki et al., 2010; Ozmert et al., 2011), Fernseh- und Computerspielkonsum (Waller et al., 2003), Mediennutzung im Allgemeinen (Must et al., 2007; Laurson, Eisenmann \& Moore, 2008; Mota et al., 2010; Mihas et al., 2009; Hardy et al., 2010).

In verschiedenen Studien zeigte sich zudem, dass der beobachtete Zusammenhang zwischen Variablen der Mediennutzung und Übergewicht bzw. Fettleibigkeit auch bei Berücksichtigung weiterer (Moderator-)Variablen (wie z. B. des sozioökonomischen Status oder der körperlichen Bewegung) bestehen bleibt, wo- 
bei die Nutzung elektronischer Bildschirmmedien allerdings wiederum nur ein weiterer Einflussfaktor im Erklärungsmodell von Adipositas oder Übergewicht ist. ${ }^{204}$ In insgesamt 66 (von 76) Querschnittsstudien sowie in 18 von 20 Längsschnittstudien wurde mindestens eine weitere Einflussvariable betrachtet, am häufigsten das Geschlecht der Kinder sowie der sozioökonomische Status oder die sportliche Betätigung bzw. Bewegung:

- das Geschlecht (Berkey et al., 2003; Proctor et al., 2003; Viner \& Cole, 2005; Hancox \& Poulton, 2006; Blair et al., 2007; Gable et al., 2007; Danner, 2008; Dubois et al., 2008; MacFarlane et al., 2009; Barnett et al., 2010; Zimmerman \& Bell, 2010; Landhuis et al., 2008; Crespo et al., 2001; Janz et al., 2002; Lowry, 2002; Giammattei et al., 2003; Storey et al., 2003; Ekelund et al., 2006; Heelan \& Eisenmann, 2006; Utter et al., 2006; Gomez et al., 2007; Beyerlein et al., 2008; Eisenmann et al., 2008; Vicente-Rodriguez et al., 2008; Wells et al., 2008; Xu et al., 2008; Khader et al., 2009; Steffen et al., 2009; Liou et al., 2010; Rivera et al., 2010; Tang et al., 2010; Perez et al., 2011; Laurson, Eisenmann, Welk, et al., 2008; Wang, Y. et al., 2007; Fulton et al., 2009; Lajous et al., 2009; Yen, C. F. et al., 2010; Lagiou \& Parava, 2008; Morales-Ruan et al., 2009; Plachta-Danielzik et al., 2011; Munakata et al., 2010; Sun et al., 2009; Janssen et al., 2004; Mendoza et al., 2007; Vandewater et al., 2004)

- das Alter (Berkey et al., 2003; Proctor et al., 2003; Henderson, 2007; Hesketh et al., 2007; Danner, 2008; MacFarlane et al., 2009; Zimmerman \& Bell, 2010; Giammattei et al., 2003; Storey et al., 2003; Ekelund et al., 2006; Utter et al., 2006; Gomez et al., 2007; Beyerlein et al., 2008; Eisenmann et al., 2008; Vicente-Rodriguez et al., 2008; Wells et al., 2008; Xu et al., 2008; Khader et al., 2009; Nagel et al., 2009; Steffen et al., 2009; Liou et al., 2010; Rivera et al., 2010; Tang et al., 2010; Perez et al., 2011; Yen, C. F. et al., 2010; Lagiou \& Parava, 2008; Morales-Ruan et al., 2009; Plachta-Danielzik et al., 2011; Janssen et al., 2004; del Mar Bibiloni et al., 2010; Mendoza et al., 2007; Vandewater et al., 2004)

- die Ethnie (Berkey et al., 2003; Henderson, 2007; Gable et al., 2007; Danner, 2008; Zimmerman \& Bell, 2010; Dubois et al., 2008; Lowry, 2002; Storey et al., 2003; Utter et al., 2006; Singh et al., 2008; Nagel et al., 2009; Perez et al., 2011; Russ et al., 2009; Vandewater et al., 2004)

- der Sozioökonomische Status (Viner \& Cole, 2005; Hancox \& Poulton, 2006; Blair et al., 2007; Delmas et al., 2007; Gable et al., 2007; Henderson, 2007; Dubois et al., 2008; Landhuis et al., 2008; Parsons et al., 2008; Storey et al.,

204 In Studien zum Zusammenhang zwischen Mediennutzung und Fettleibigkeit bzw. Übergewicht wurden im Vergleich zu Studien zur Schulleistung oder zum Gewaltverhalten deutlich häufiger eine Vielzahl von Einflussvariablen berücksichtigt. 
2003; Ekelund et al., 2006; Utter et al., 2006; Gomez et al., 2007; Laxmaiah et al., 2007; Singh et al., 2008; Vicente-Rodriguez et al., 2008; Wells et al., 2008; Garcinuno et al., 2010; Rivera et al., 2010; Tang et al., 2010; Tremblay \& Willms, 2003; Stettler et al., 2004; Kautiainen et al., 2005; Yen, C. F. et al., 2010; Lagiou \& Parava, 2008; Russ et al., 2009; Plachta-Danielzik et al., 2011; Lazarou \& Soteriades, 2010; Aucote \& Cooper, 2009; Bishwalata et al., 2010; Mendoza et al., 2007; Vandewater et al., 2004; del Mar Bibiloni et al., 2010; Khader et al., 2009)

- das Bildungsniveau (Proctor et al., 2003; Gable et al., 2007; Henderson, 2007; MacFarlane et al., 2009; Zimmerman \& Bell, 2010; Dubois et al., 2008; Toschke et al., 2008; Wells et al., 2008; Khader et al., 2009; Garcinuno et al., 2010; Wake et al., 2003; del Mar Bibiloni et al., 2010)

- Stadt/Land (Singh et al., 2008; Liou et al., 2010; Lazarou \& Soteriades, 2010)

- die Familienstruktur (Tremblay \& Willms, 2003; Lagiou \& Parava, 2008; Wake et al., 2003)

- die sportliche Betätigung/körperliche Aktivität (Proctor et al., 2003; Viner \& Cole, 2005; Blair et al., 2007; Delmas et al., 2007; Henderson, 2007; Dubois et al., 2008; MacFarlane et al., 2009; Barnett et al., 2010; Parsons et al., 2008; Crespo et al., 2001; Janz et al., 2002; Storey et al., 2003; Utter et al., 2006; Laxmaiah et al., 2007; Xu et al., 2008; Tremblay \& Willms, 2003; Stettler et al., 2004; Kautiainen et al., 2005; Wake et al., 2003; Vandewater et al., 2004; Burke et al., 2006; Carvalhal et al., 2006; Kuriyan et al., 2007; Eisenmann et al., 2008; Singh et al., 2008; Jackson, D. M. et al., 2009; de Gouw et al., 2010; Garcinuno et al., 2010; Liou et al., 2010; Oliver et al., 2010; Papandreou et al., 2010; Rivera et al., 2010; Lehto et al., 2011; Perez et al., 2011; Wang, Y. et al., 2007; Yen, C. F. et al., 2010; Lagiou \& Parava, 2008; Danielsen et al., 2011; Lazarou \& Soteriades, 2010; Munakata et al., 2010; Sun et al., 2009; del Mar Bibiloni et al., 2010; Schneider, M. et al., 2007)

- die Ernährung (Berkey et al., 2003; Proctor et al., 2003; Gable et al., 2007; Parsons et al., 2008; Crespo et al., 2001; Lowry, 2002; Giammattei et al., 2003; Storey et al., 2003; Gomez et al., 2007; Kuriyan et al., 2007; Beyerlein et al., 2008; Chang \& Nayga, 2009; de Gouw et al., 2010; Li, X. et al., 2010; Liou et al., 2010; Papandreou et al., 2010; Rosenkranz et al., 2010; Tang et al., 2010; Lehto et al., 2011; Wang, Y. et al., 2007; Danielsen et al., 2011; Lazarou \& Soteriades, 2010; Sun et al., 2009; Wake et al., 2003; Janssen et al., 2004; Bishwalata et al., 2010; Burke et al., 2006)

- andere Freizeitaktivitäten (Delmas et al., 2007; Gable et al., 2007; Khader et al., 2009; Liou et al., 2010; Oliver et al., 2010; Tang et al., 2010; Janssen et 
al., 2004; Burke et al., 2006; Vandewater et al., 2004; Schneider, M. et al., 2007)

- der Schlaf (Kuriyan et al., 2007; Xu et al., 2008; Liou et al., 2010; Lehto et al., 2011; Lagiou \& Parava, 2008; Lazarou \& Soteriades, 2010; del Mar Bibiloni et al., 2010)

- das Rauchen (Parsons et al., 2008; Ekelund et al., 2006; Morales-Ruan et al., 2009; del Mar Bibiloni et al., 2010)

- der Alkoholkonsum (Parsons et al., 2008; Morales-Ruan et al., 2009; del Mar Bibiloni et al., 2010)

- das Taschengeld (Khader et al., 2009; Xu et al., 2008)

- die (Geschlechts-)reife (Berkey et al., 2003; Delmas et al., 2007; Henderson, 2007; Parsons et al., 2008; Ekelund et al., 2006; Kautiainen et al., 2005)

- das Wissen über Fettleibigkeit (Bishwalata et al., 2010)

- die Kardiovaskuläre Fitness (Schneider, M. et al., 2007)

- der BMI Mutter/Vater/Eltern (Proctor et al., 2003; Viner \& Cole, 2005; Hancox \& Poulton, 2006; Blair et al., 2007; Landhuis et al., 2008; Parsons et al., 2008; MacFarlane et al., 2009; Zimmerman \& Bell, 2010; Beyerlein et al., 2008; Toschke et al., 2008; Wells et al., 2008; Khader et al., 2009; Nagel et al., 2009; Steffen et al., 2009; Garcinuno et al., 2010; Li, X. et al., 2010; Liou et al., 2010; Oliver et al., 2010; Papandreou et al., 2010; Rosenkranz et al., 2010; Plachta-Danielzik et al., 2011; Wake et al., 2003; Bishwalata et al., 2010)

- das Rauchen der Mutter/in der Schwangerschaft (Blair et al., 2007; Toschke et al., 2008; Nagel et al., 2009; Stettler et al., 2004)

- das Geburtsgewicht (Viner \& Cole, 2005; Danner, 2008; Ekelund et al., 2006; Wells et al., 2008; Nagel et al., 2009; Li, X. et al., 2010)

- die Gewichtszunahme in den ersten beiden Lebensjahren (Beyerlein et al., 2008; Toschke et al., 2008; Li, X. et al., 2010)

- das Stillen (Blair et al., 2007; Toschke et al., 2008; Nagel et al., 2009; Li, X. et al., 2010; Papandreou et al., 2010)

- die Arbeit der Mutter (Stettler et al., 2004)

Legt man die einzige Metaanalyse von Marshall und Kollegen (Marshall et al., 2004), die für die Jahre 2001 bis 2011 aufgefunden wurde, zugrunde, muss von einem kleinen kausalen Zusammenhang zwischen der Nutzung digitaler Medien und Fettleibigkeit bzw. Übergewicht ausgegangen werden. So berichten die Autoren in ihrer Analyse von 107 Querschnitts-, Längsschnitt- und Interventionsstudien aus Amerika, Asien, Europa und Australien zum Zusammenhang der Fernseh- und Video- bzw. Computerspielnutzung mit Körperfettgehalt und Bewegungsarmut eine mittlere Effektstärke zwischen Fernsehnutzung und Körperfettgehalt von $r=.08$ (CI.06 bis.08; $k=52$ Samples, $n=44.707$ ) sowie eine mitt- 
lere Effektstärke zwischen Computerspielnutzung und Körperfettgehalt von $r=$ 13 (CI -.05 bis. 19; $k=6$ Samples, $n=1.722$ ). Darüber hinaus wurde zwischen Fernsehnutzung und körperlicher Bewegung eine mittlere Effektstärke von $r=$-. 13 ( $C I-.08$ bis $-.11 ; k=39$ Samples, $n=141.505$ ) sowie zwischen Computerspielnutzung und körperlicher Bewegung eine mittlere Effektstärke von $r=-.14$ ( $C I-.08$ bis $-.13 ; k=10$ Samples, $n=119.942$ ) ermittelt. Die deutlichsten $\mathrm{Zu}-$ sammenhänge zwischen Fernsehnutzung und Körperfettgehalt zeigten sich dabei für Studien mit Studienteilnehmern im Alter von 0-6 Jahren $(r=.19$, CI.09 bis. 20; $k=4$ Samples, $n=2.047)$. Die deutlichsten Zusammenhänge zwischen Fernsehnutzung und körperlicher Bewegung zeigten sich hingegen für Studien mit Studienteilnehmern im Alter von 13-18 Jahren $(r=-.15, C I-.09$ bis $-.14 ; k=16$ Samples, $n=14.630$ ). Insgesamt folgern die Autoren, dass bei 99 Prozent Varianzaufklärung des Körperfettgehalts durch andere Faktoren neben der Mediennutzung, die klinische Bedeutsamkeit des Zusammenhangs zwischen Mediennutzung und Fettleibigkeit in Frage gestellt werden muss.

"It is concluded that a statistically small relationship exists between TV viewing and body fatness among children and youth although the magnitude of the relationship suggests that we should be cautious about the clinical relevance of this finding. The strength of this relationship remains virtually unchanged even after correcting for common sources of bias known to impact study outcomes." (Marshall et al., 2004, S. 1244)

Trotz dieser teilweise geringen Effekte weisen Marshall und Kollegen (Marshall et al., 2004) jedoch explizit auf die Bedeutsamkeit von Interventionsprogrammen mit der Zielsetzung der Medienkonsumreduktion und Bewegungssteigerung für die Adipositasprävention hin. So konnte in einigen Studien durch eine Reduktion des Medienkonsums (Robinson, 2001; Epstein et al., 2008), durch eine Steigerung der körperlichen Bewegung (Mhurchu et al., 2008) oder durch eine Kombination aus beidem (Plachta-Danielzik et al., 2008; Salmon et al., 2008; Epstein et al., 2011) das Körpergewicht der teilnehmenden Schülerinnen und Schüler positiv beeinflusst werden. ${ }^{205}$

205 In den Studien von Robinson und Kollegen (Robinson et al., 2010) bzw. Taveras und Kollegen (Taveras et al., 2011) konnte bei ähnlichem Anliegen allerdings lediglich der Fernsehkonsum, nicht jedoch der Body Mass Index (BMI) reduziert werden. In der Studie von Mhurchu und Kollegen (Mhurchu et al., 2008) bestand die Bewegungssteigerung im Spielen von aktivitätsfördernden Computerspielen (Eye-Toy). 


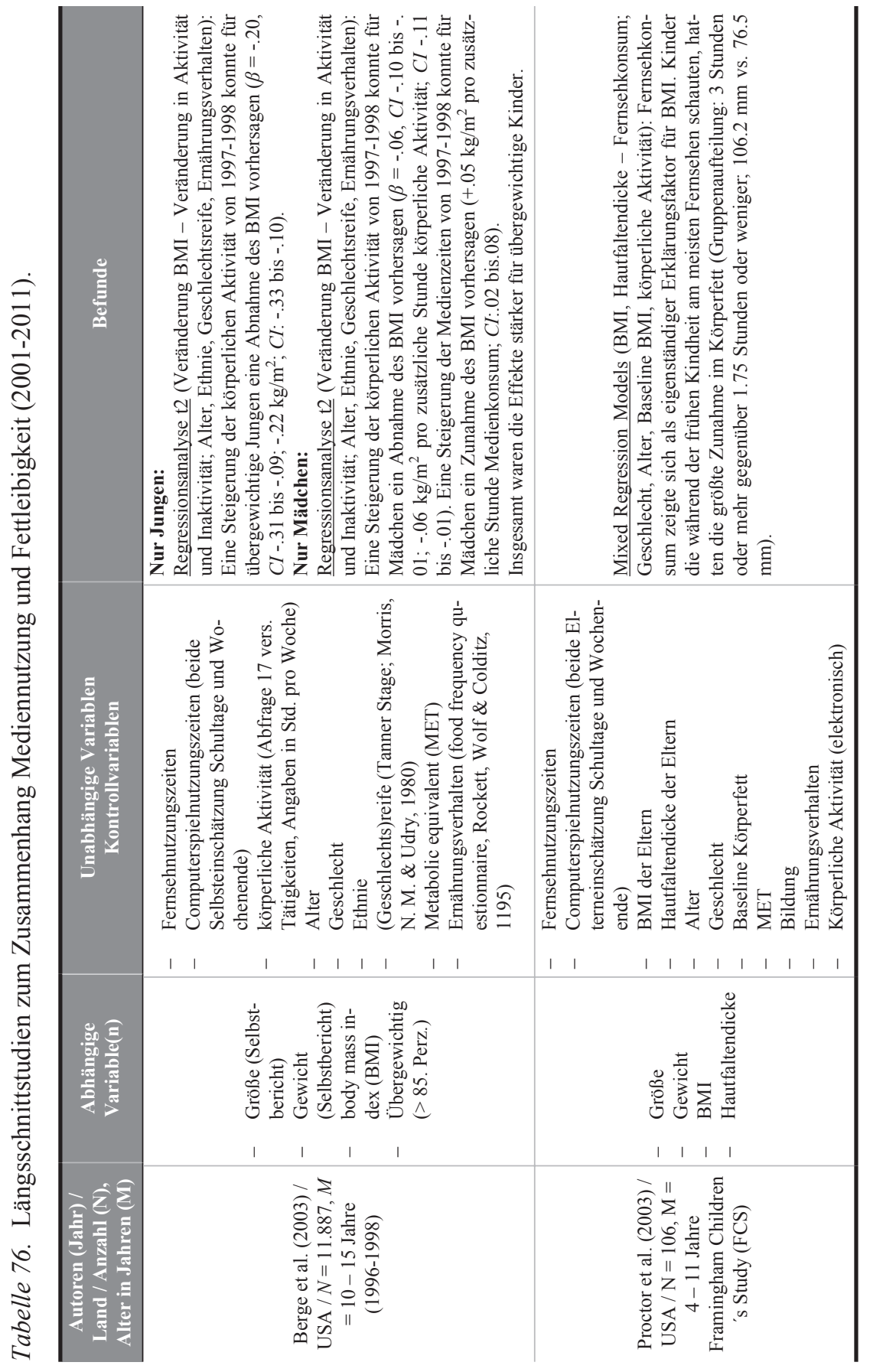




\begin{tabular}{|c|c|c|c|}
\hline हू & 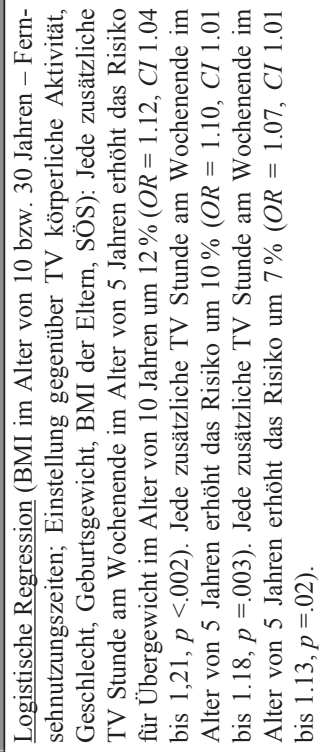 & 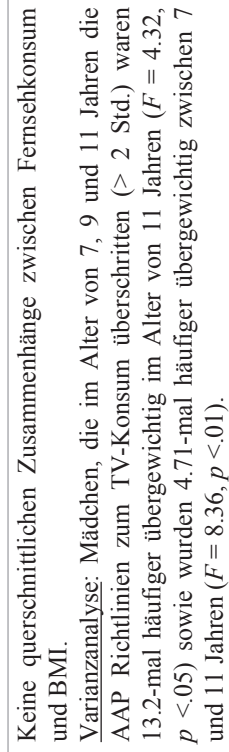 & 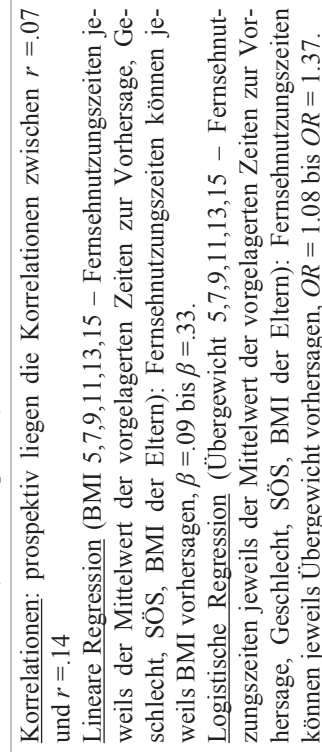 \\
\hline 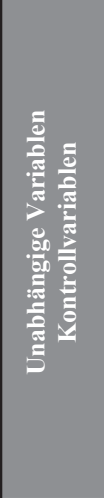 & 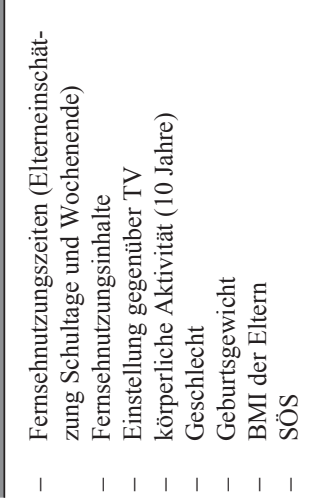 & 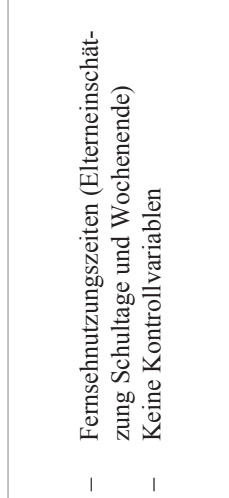 & 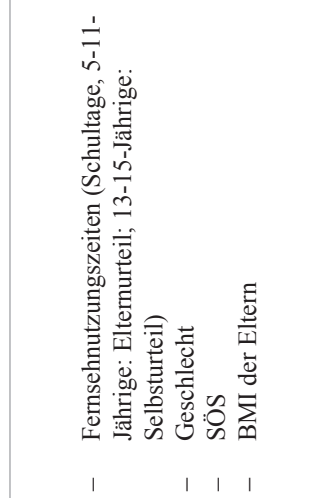 \\
\hline 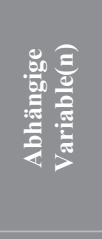 & 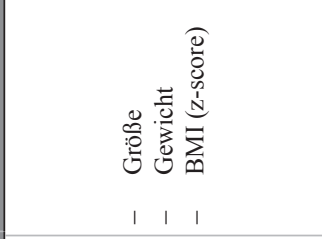 & 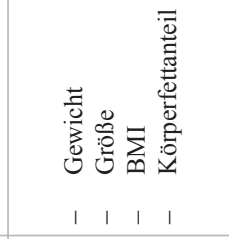 & 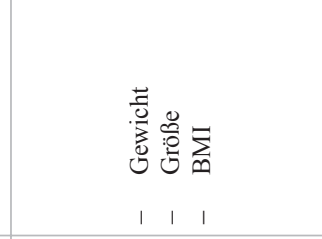 \\
\hline 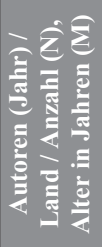 & 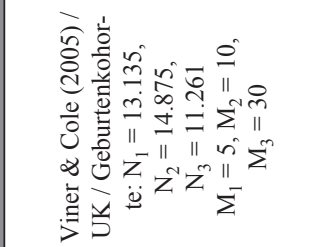 & 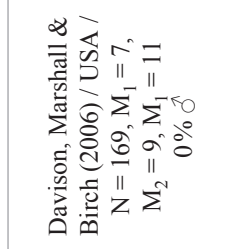 & 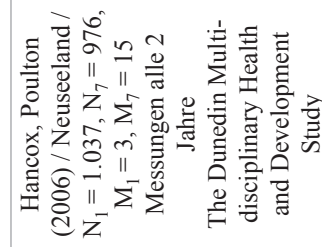 \\
\hline
\end{tabular}




\begin{tabular}{|c|c|c|}
\hline & 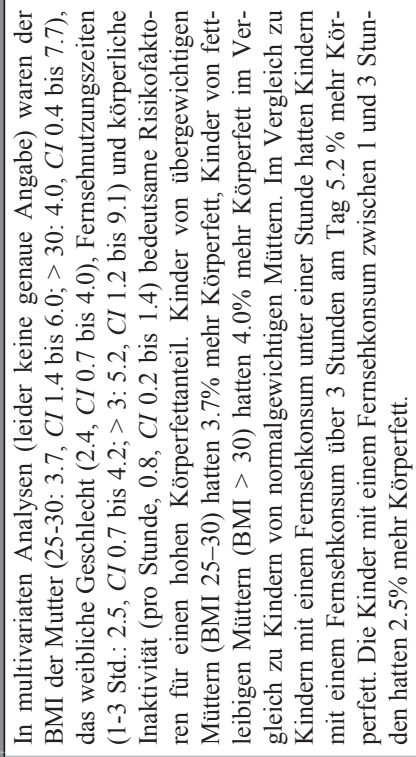 & 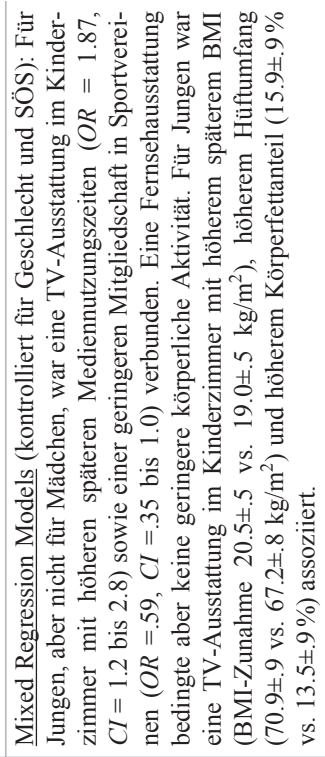 \\
\hline 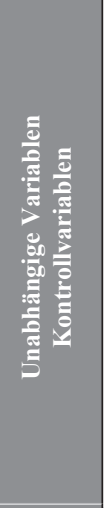 & 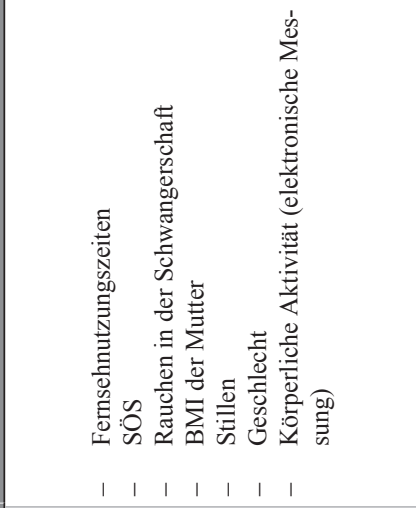 & 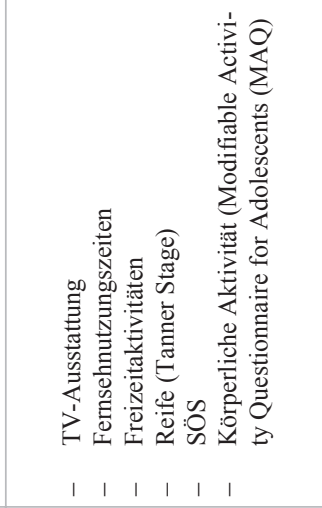 \\
\hline 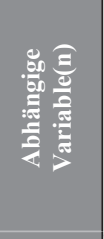 & 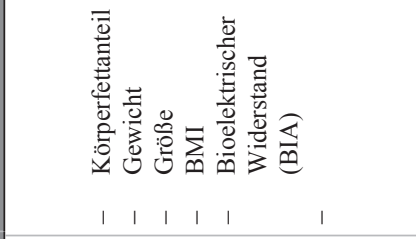 & 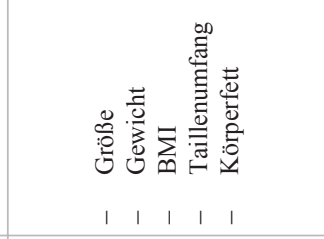 \\
\hline 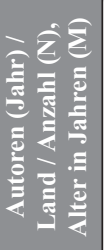 & 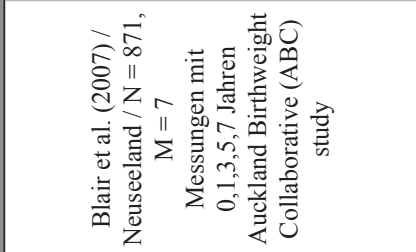 & 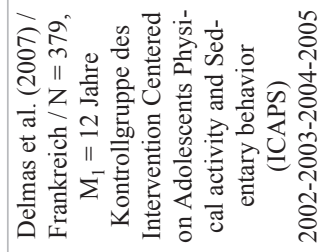 \\
\hline
\end{tabular}




\begin{tabular}{|c|c|c|}
\hline 营 & 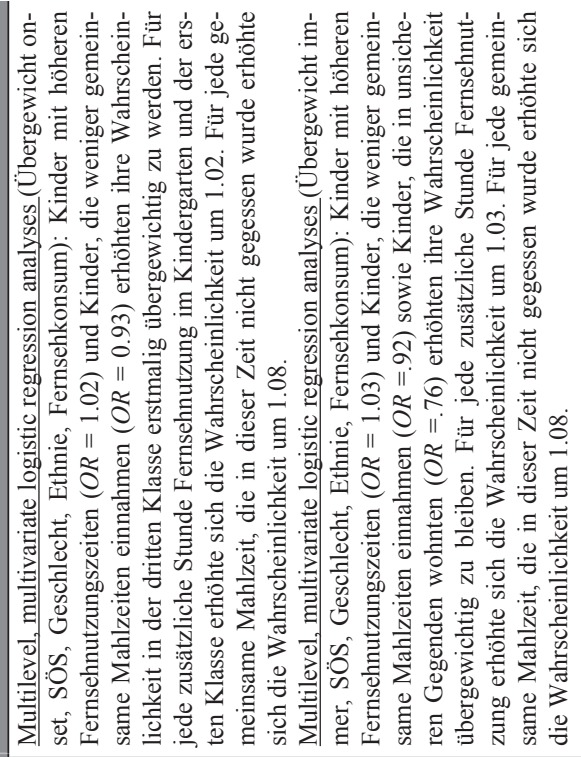 & 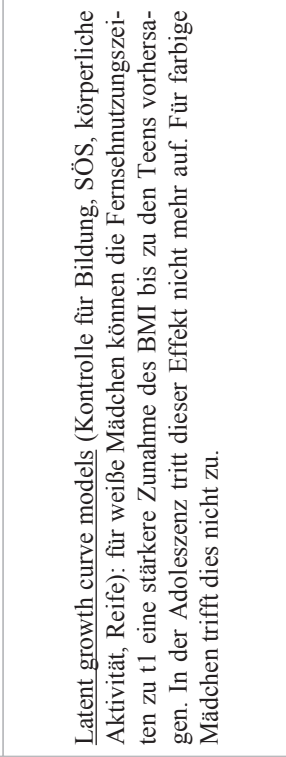 \\
\hline 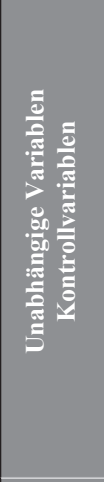 & 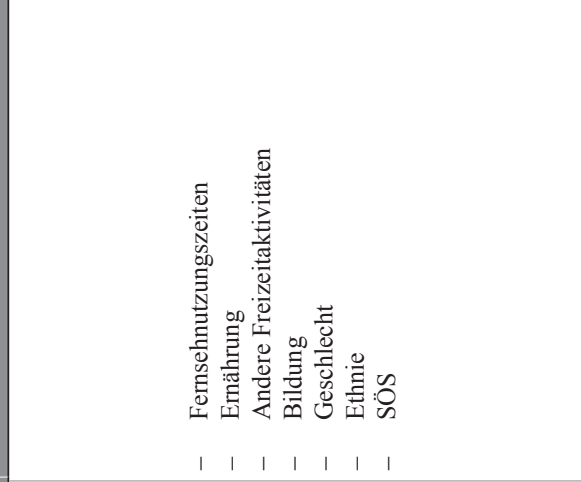 & 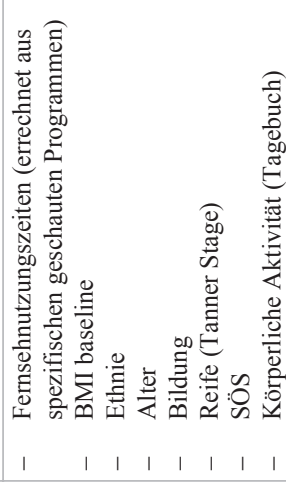 \\
\hline 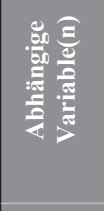 & 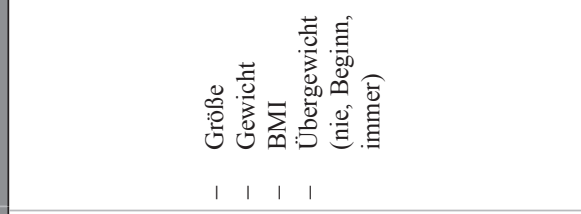 & 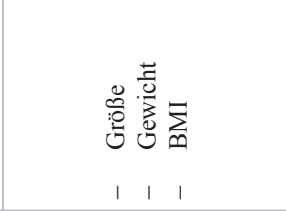 \\
\hline 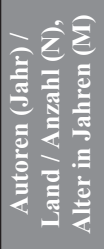 & 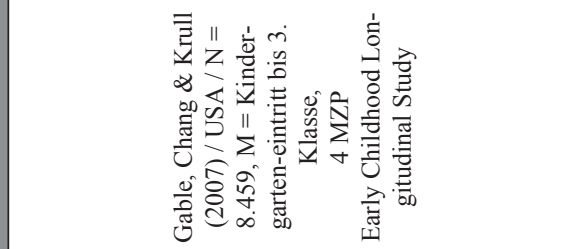 & 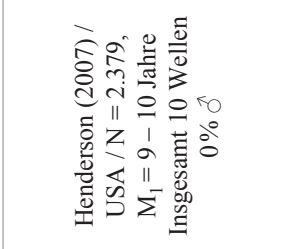 \\
\hline
\end{tabular}




\begin{tabular}{|c|c|c|}
\hline 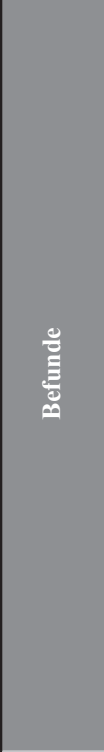 & 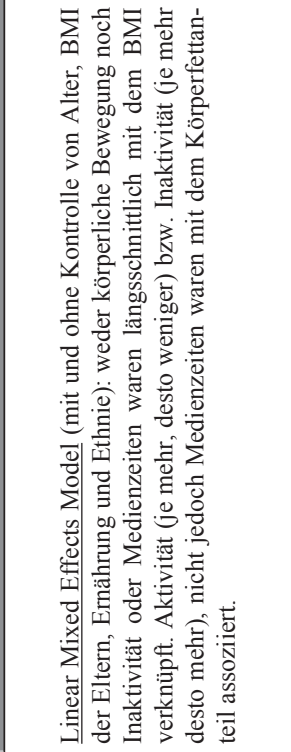 & 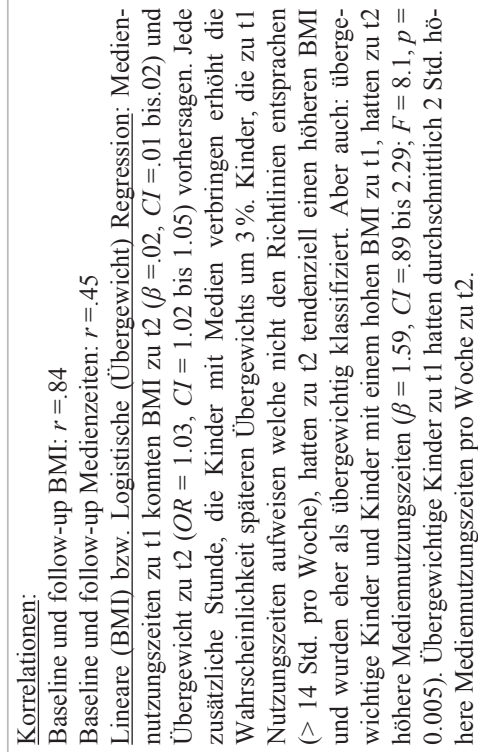 \\
\hline 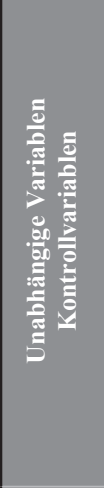 & 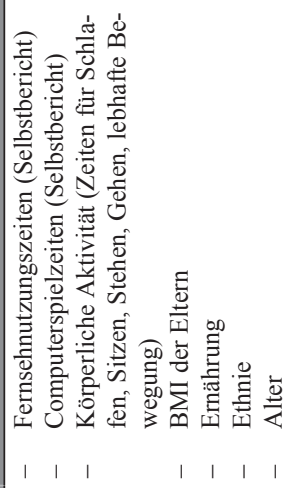 & 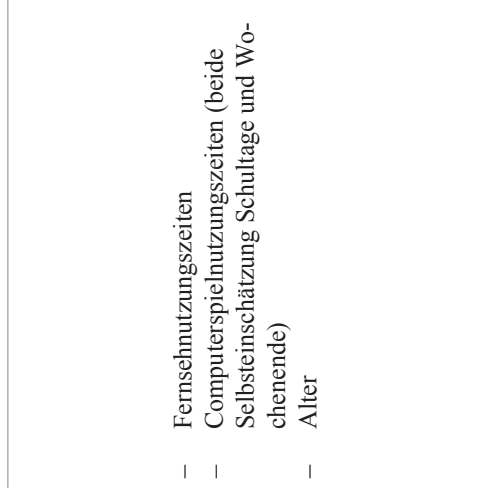 \\
\hline 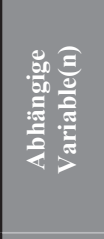 & 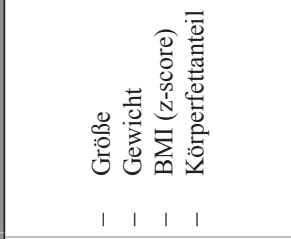 & 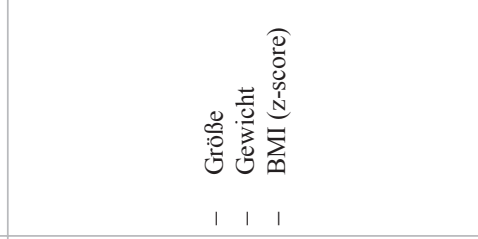 \\
\hline 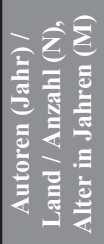 & 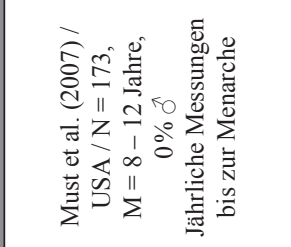 & 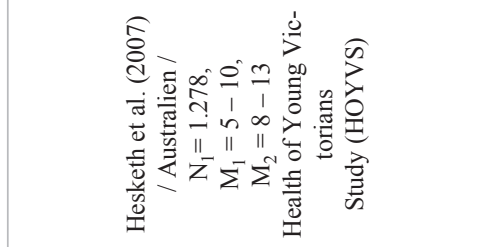 \\
\hline
\end{tabular}




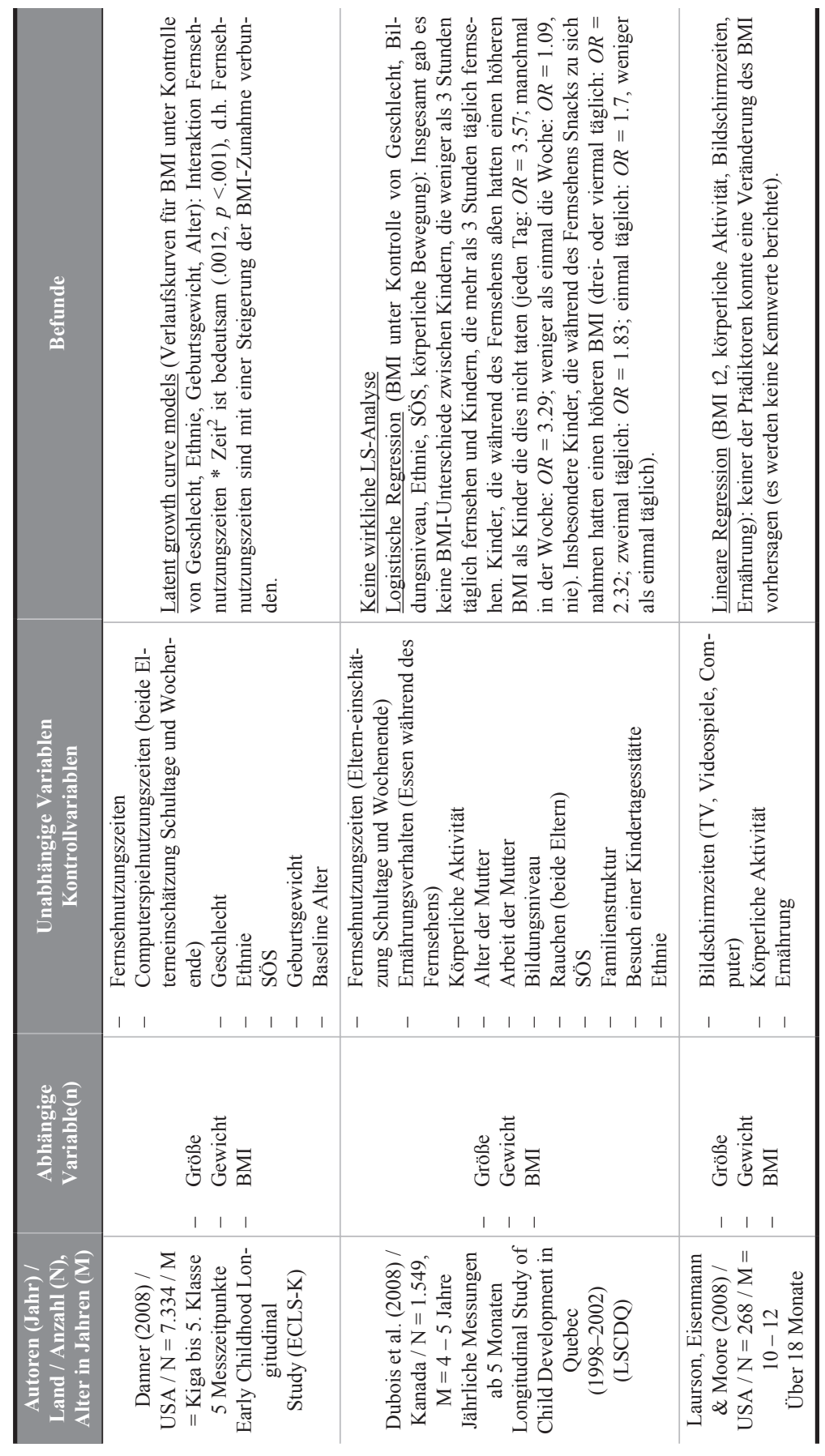




\begin{tabular}{|c|c|c|}
\hline हूँ & 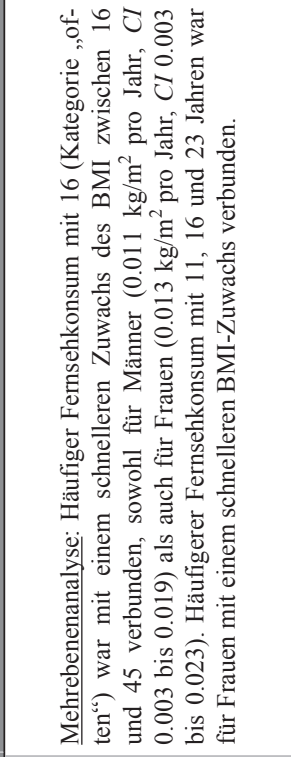 & 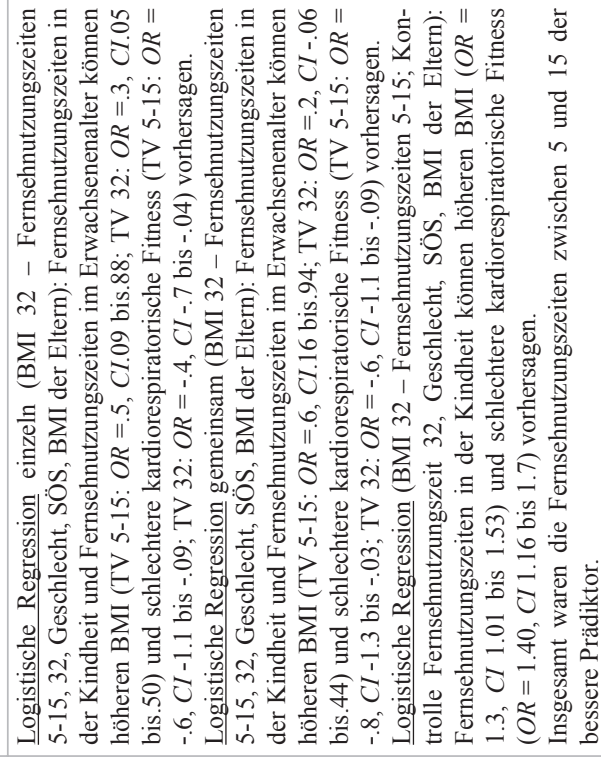 \\
\hline 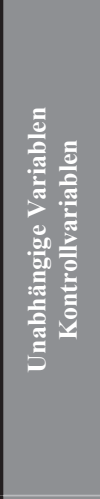 & 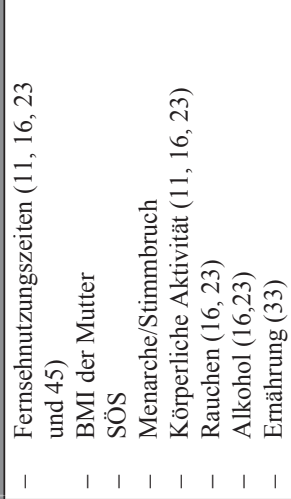 & 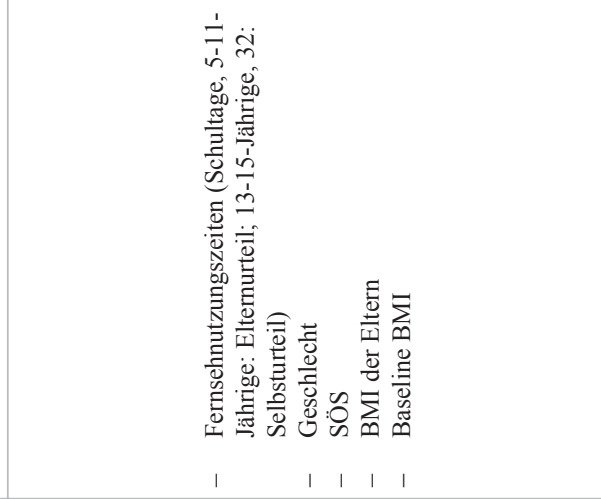 \\
\hline 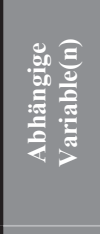 & 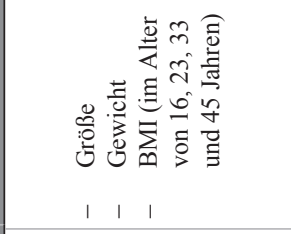 & 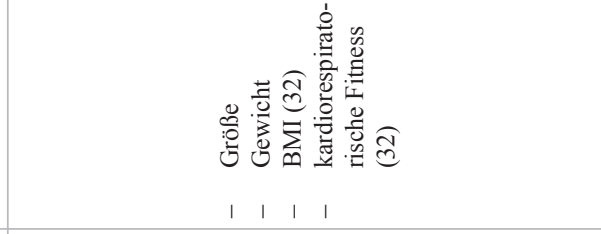 \\
\hline 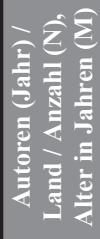 & 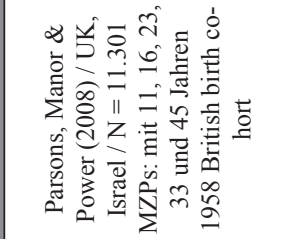 & 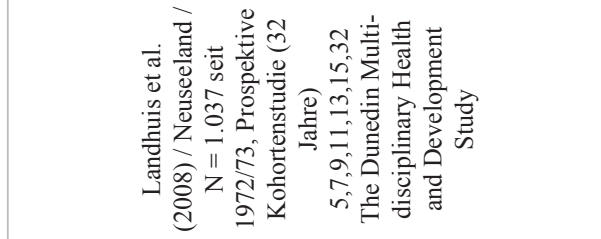 \\
\hline
\end{tabular}




\begin{tabular}{|c|c|c|c|}
\hline 离 & 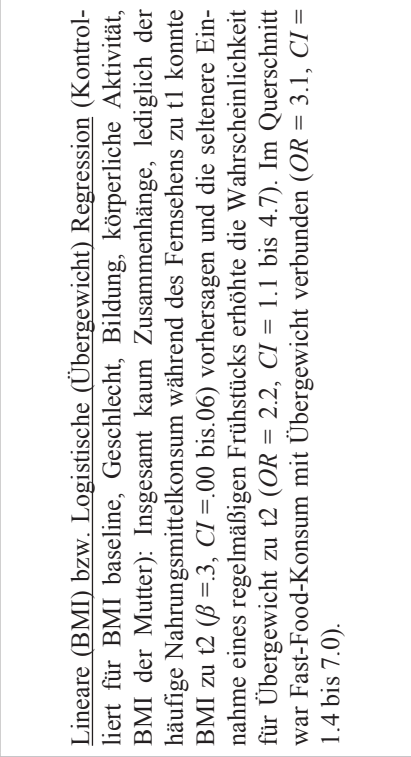 & 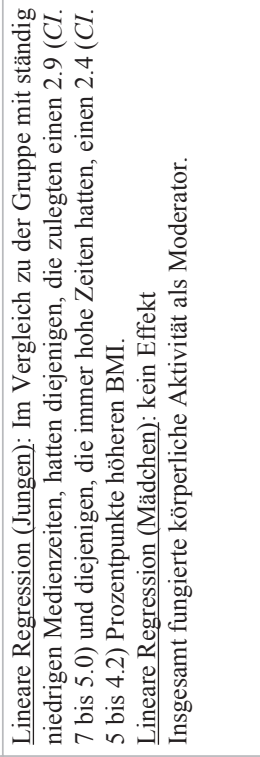 & 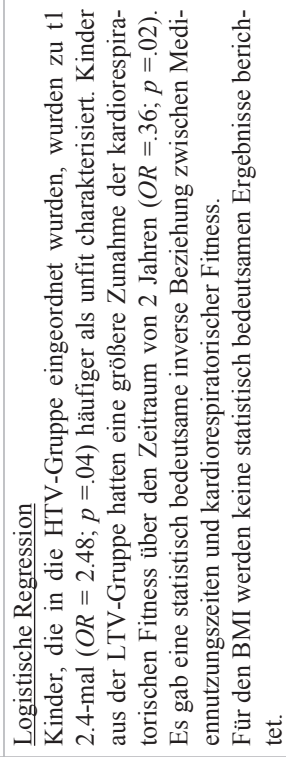 \\
\hline 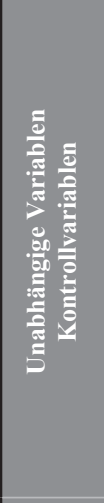 & 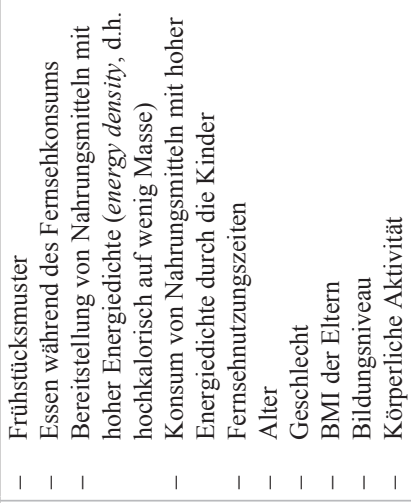 & 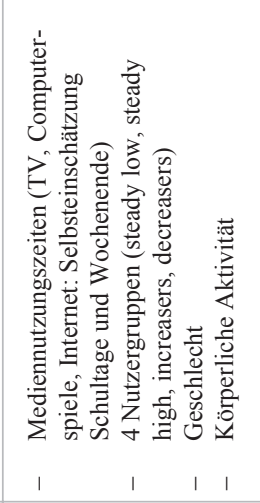 & 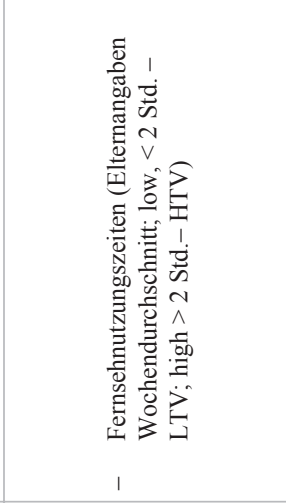 \\
\hline 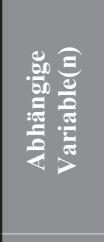 & 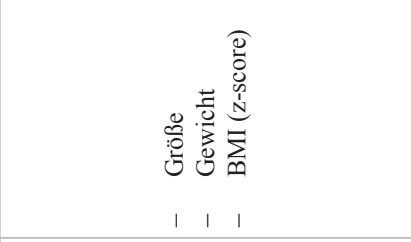 & 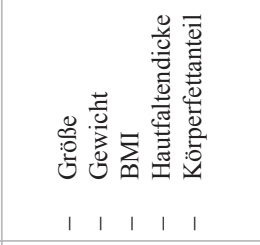 & 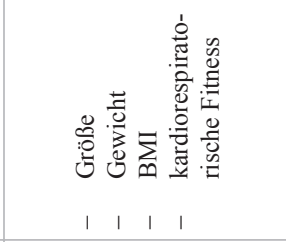 \\
\hline 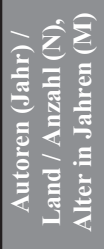 & 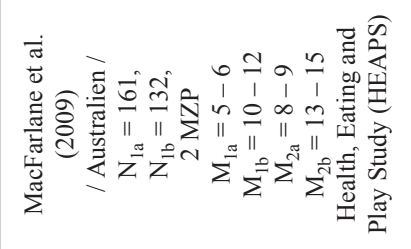 & 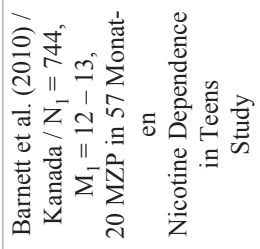 & 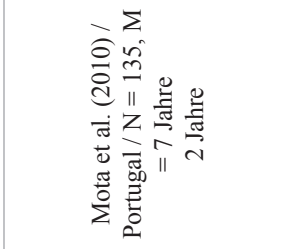 \\
\hline
\end{tabular}




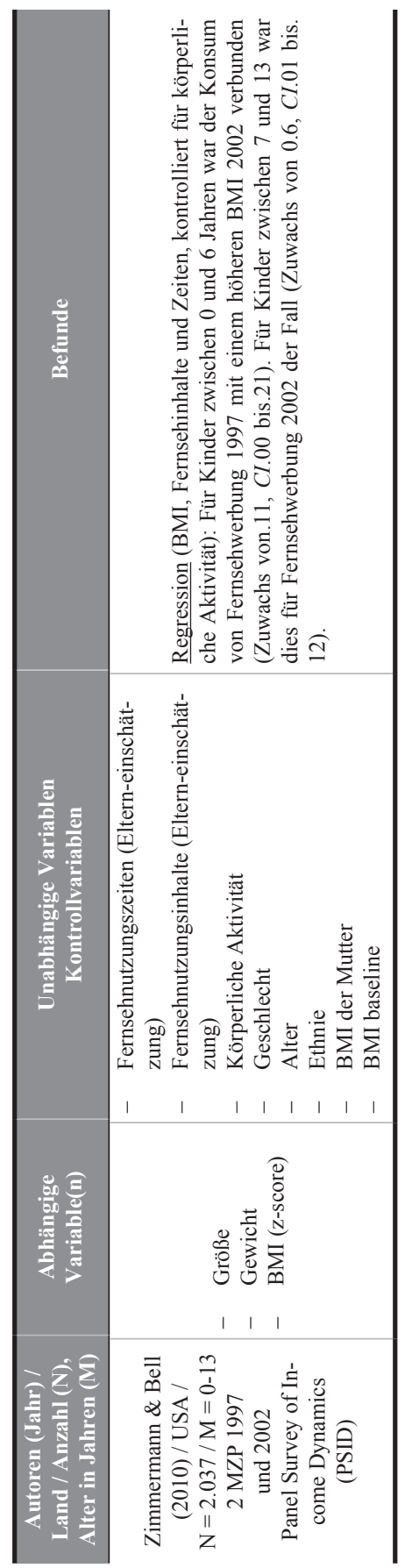


6.1.2 Erklärungsmodelle zum Zusammenhang Mediennutzung und Fettleibigkeit

Zur Erklärung des genauen Wirkzusammenhangs zwischen der Nutzung elektronischer Bildschirmmedien und Übergewicht bzw. Fettleibigkeit oder Adipositas bei Kindern und Jugendlichen können die folgenden theoretischen Annahmen angeführt werden, welche sich in die drei Hauptthemengebiete Bewegungsmangel, (durch Medien beeinflusstes) Gesundheits- und Ernährungsverhalten sowie Schlafmangel einteilen lassen (vgl. Kaiser Family Foundation, 2004):

\section{Bewegungsmangel:}

- Die Zeit, die Kinder und Jugendliche mit elektronischen Bildschirmmedien verbringen, verdrängt Zeit, die mit körperlicher Betätigung verbracht werden könnte.

- Die Motivation zu körperlicher Bewegung nimmt mit wachsendem Bildschirmmedienkonsum ab.

\section{Ernährung:}

- Die Nahrungsmittelwerbung, mit der Kinder und Jugendliche täglich medial konfrontiert sind, führt zu einer ungesunden Ernährungswahl.

- Die Cross-Promotion von Nahrungsmitteln und TV- sowie Filmfiguren animiert Kinder und Jugendliche zum Konsum hochkalorischer Nahrungsmittel.

- Kinder und Jugendliche haben ein ausschweifendes Snackverhalten beim Medienkonsum und konsumieren ungesündere Nahrungsmittel, wenn vor dem Fernseher gegessen wird.

- Fernseh- und Videokonsum führt zu einem verringertem Umsatz (metabolic rate), der unter dem des Schlafes liegt.

- Die Darstellungen von Nahrung und Körpergewicht in den Unterhaltungsmedien führen zu ungesünderen Ernährungsweisen bei Kindern und Jugendlichen.

\section{Schlafmangel:}

- Da exzessiver Medienkonsum mit verkürzten Schlafenszeiten einhergeht und diese wiederum mit Übergewicht und Adipositas zusammenhängen, erhöhen Kinder und Jugendliche durch einen hohen Medienkonsum das Risiko von Übergewicht und Adipositas.

Bewegungsmangel. Besonders häufig wird zur Erklärung des Wirkzusammenhangs zwischen der Nutzung elektronischer Bildschirmmedien und Übergewicht bzw. Fettleibigkeit bei Kindern und Jugendlichen ein Bewegungsmangel bzw. eine geringe körperliche Aktivität angeführt (vgl. Crespo et al., 2001; Storey et al., 2003; Janz et al., 2002; Utter et al., 2006; Laxmaiah et al., 2007; Singh et al., 
2008; Xu et al., 2008; de Gouw et al., 2010; Liou et al., 2010; Oliver et al., 2010; Papandreou et al., 2010; Rivera et al., 2010; Lehto et al., 2011; Perez et al., 2011; Tremblay \& Willms, 2003; Stettler et al., 2004; Kautiainen et al., 2005; Wang, Y. et al., 2007; Yen, C. F. et al., 2010; Lagiou \& Parava, 2008; Danielsen et al., 2011). Nicht zuletzt, da in Interventionsprogrammen in denen sowohl der Medienkonsum reduziert als auch die Bewegung explizit gesteigert wurde, positive Effekte auf das Körpergewicht erzielt werden konnten (vgl. Epstein et al., 2008; Plachta-Danielzik et al., 2008; Salmon et al., 2008). So lag in der Metaanalyse von Marshall und Kollegen (Marshall et al., 2004) der berichtete negative $\mathrm{Zu}$ sammenhang von Mediennutzung und körperlicher Betätigung für das Fernsehen bei $r=-.096$ und für das Computerspielen bei $r=-.104$.

Stettler und Kollegen (Stettler et al., 2004) konnten beispielsweise in einer Studie mit 872 Schweizer Grundschülerinnen und Grundschülern der ersten bis dritten Klasse bei Kontrolle der Fernsehnutzung und weiterer wichtiger soziodemografischer Merkmale der Schülerinnen und Schüler einen eindeutigen Zusammenhang zwischen dem Spielen von elektronischen Spielen und dem BMI finden. Das Risiko der untersuchten Erst- bis Drittklässler für Übergewicht stieg mit jeder zusätzlichen täglichen Computerspielstunde auf das Doppelte an (Stettler et al., 2004). Zudem wird insbesondere bei Intensivspielern eine ungünstige Energiebilanz sowie eine Erhöhung des Körperfettanteils und damit langfristig Übergewicht befürchtet (Cordes \& Miller, 2001). Myrtek und Scharff (2000) untersuchten mit jeweils 100 11- und 15-jährigen Schülerinnen und Schülern die Auswirkungen der Mediennutzung insbesondere auf die körperliche und emotionale Befindlichkeit. Die Autoren konnten mithilfe einer physiologischen Messung der Bewegungsaktivität sowie der Herzfrequenz eine deutlich geringere körperliche Aktivität sowie das Meiden stärkerer Belastungen, wie z.B. Treppen steigen oder Fahrrad fahren, bei den Vielsehern nachweisen (Myrtek \& Scharff, 2000). Darüber hinaus hatte diese Gruppe weniger soziale Kontakte, konnte weniger gut mit Stress umgehen und war insgesamt in ihren kommunikativen Fähigkeiten eingeschränkt.

Ernährung. Der Einfluss der Medien und insbesondere der Werbung auf eine ungesunde Ernährungswahl, d. h. besonders hochkalorische aber ansonsten nähwertarme Nahrungsmittel, wurde in zahlreichen Überblicksartikeln und Reviews, hier insbesondere auch im Hinblick auf daraus zu ziehende politische Folgerungen und Handlungsrichtlinien zur Vermeidung von Übergewicht bei Kindern und Jugendlichen, zusammengefasst (Hastings, G. et al., 2003; Kunkel et al., 2004; Livingstone, 2005; McGinnis, Gootman \& Kraak, 2005; Hastings, G., McDermott, Angus, Stead \& Thomson, 2007). Hastings und Kollegen (Hastings, G. et al., 2007) resümieren die Studienlage zur allgemeinen Werbewirkung auf die Ernährung von Kindern und Jugendlichen wie folgt: 
“Food adverts are among [...children's] favourite types of advertising, with the most popular being for chocolate, sweets, soft drinks and other foods high in fat, sugar and salt (e.g. snacks and fizzy drinks). Evidence from more complex studies (capable of establishing causality) shows that this promotional activity is having an effect on children. Whilst it does not seem to influence their general perceptions of what constitutes a healthy diet, it can have an effect on more specific types of nutritional knowledge. Beyond knowledge, food promotion influences children's food preferences, and encourages them to ask their parents to purchase foods they have seen advertised. Food promotion has also been shown to influence children's consumption and other diet-related behaviours and outcomes. These effects are significant, independent of other influences and operate at both brand and category level." (Hastings, G. et al., 2007, S. 2)

Ferner fassen McGinnis und Kollegen (McGinnis et al., 2005) die Forschungsbefunde für das speziellere Feld der Wirkung von TV-Werbung auf kindliches Ernährungsverhalten folgendermaßen zusammen:

"[...] Among many factors, food and beverage marketing influences the preferences and purchase requests of children, influences consumption at least in the short term, is a likely contributor to less healthful diets, and may contribute to negative diet-related health outcomes and risks among children and youth. The literature indicates relationships among marketing, dietary precursors, diets, diet-related health, and, in particular, adiposity (body fatness).

[...] Given the findings from the systematic evidence review of the influence of marketing on the precursors of diet, and given the evidence from content analyses that the preponderance of television food and beverage advertising relevant to children and youth promotes high-calorie and low-nutrient products, it can be concluded that television advertising influences children to prefer and request high-calorie and low-nutrient foods and beverages.

[...] The association between adiposity and exposure to television advertising remains after taking alternative explanations into account, but the research does not convincingly rule out other possible explanations for the association; therefore, the current evidence is not sufficient to arrive at any finding about a causal relationship from television advertising to adiposity. It is important to note that even a small influence, aggregated over the entire population of American children and youth, would be consequential in impact." (McGinnis et al., 2005, S. 8f) 
Den Nachweis einer Beeinflussung des Umsatzes (metabolic rate) durch mediale Tätigkeiten erbrachten Klesges und Kollegen (Klesges, Shelton \& Klesges, 1993) für das Fernsehen in einem Experiment mit 31 8- bis 12-jährigen Kindern, wobei die Hälfte dieser übergewichtig war. Als Ergebnis zeigte sich, dass der Umsatz während des TV-Konsums geringer war als während einer Ruhezeit. Darüber hinaus war dieser Unterschied bei übergewichtigen Kindern größer. Zahlreiche andere Studien konnten diesen Effekt allerdings nicht bestätigen (vgl. Kaiser Family Foundation, 2004), weswegen diese Annahme zumindest als noch nicht geklärt betrachtet werden muss.

Schlafmangel. Insbesondere im Grundschulalter werden Einschlafprobleme sowie Schlafstörungen oder Schlafmangel mit einer Vielzahl psychischer, sozialer oder medizinischer Probleme assoziiert (motorische und kognitive Leistungen, vgl. Pilcher \& Huffcutt, 1996; Aufmerksamkeits- und Schulleistungsprobleme, vgl. Owens et al., 1999; Verhaltensprobleme, vgl. Stein, Mendelsohn, Obermeyer, Amromin \& Benca, 2001). So werden verkürzte Schlafzeiten auch als Risikofaktor von Übergewicht und Adipositas betrachtet (vgl. Kuriyan et al., 2007; Heins et al., 2007; Wells et al., 2008; Liou et al., 2010; Monasta et al., 2010, siehe Abbildung 78). Auf der anderen Seite werden wiederholt Zusammenhänge zwischen einer exzessiven Mediennutzung und Einschlafproblemen (vgl. Owens et al., 1999) bzw. reduzierten Schlaf- und Erholungszeiten (vgl. Gillespie, 2002; Van den Bulk, 2004) berichtet. Letzteres ist bei einer Begrenzung des Tages auf 24 Stunden augenscheinlich: Bei exzessiver Mediennutzung und gleich bleibenden nichtmedialen Freizeit- sowie sonstigen Aktivitäten kann diese lediglich dadurch kompensiert werden, indem erst später zu Bett gegangen wird. ${ }^{206}$ Dies konnte insbesondere für die Fernsehnutzung mehrfach gezeigt werden (vgl. Adam, Snell \& Pendry, 2007; Crönlein et al., 2007; Gaina et al., 2007; Heins et al., 2007; Li, S. et al., 2007). Crönlein und Kollegen (Crönlein et al., 2007) ergänzten in ihrer Studie mit 137 im Mittel 13-jährigen Schülerinnen und Schülern diesen Befund um die Wichtigkeit, neben der Quantität der Mediennutzung auch die konsumierten Inhalte zu betrachten: Vor allem bei Jungen hingen zwar längere Fernsehzeiten insgesamt mit verkürzten Schlafenszeiten zusammen, der häufige Konsum von Gruselfilmen führte jedoch für beide Geschlechter zu einem gestörteren Einschlafen. Darüber hinaus beeinflusste dieses Filmgenre auch das

206 Zudem konnte vielfach die Bedeutung der eigenen Mediengeräte im Kinderzimmer für verringerte Schlafenszeiten, Schlafstörungen und die Schlafqualität insgesamt gezeigt werden (Owens et al., 1999; Li, S. et al., 2007; Lehmkuhl, Wiater, Mitschke \& FrickeOerkermann, 2008). 
subjektive Empfinden des Schlafens („Gefühl nicht geschlafen zu haben“). ${ }^{207}$ Heins und Kollegen (Heins et al., 2007) berichten in ihrer Querschnittsbefragung mit 1.933 im Mittel 10-jährigen Grundschülerinnen und Grundschülern ähnliche Ergebnisse. So hing eine Schlafenszeit nach $21 \mathrm{Uhr}$ mit einem höheren Alter, männlichem Geschlecht, älteren Geschwistern, der Schullokalisation in der Stadt, dem Besitz eines eigenen Handys und einem Fernseh- und Computerkonsum von mehr als 3 Stunden täglich zusammen. Verkürzte Schlafzeiten bei einer intensiven Computer(spiel)nutzung konnte für Jugendliche in weiteren Studien nachgewiesen werden (vgl. Griffiths, Davies \& Chappell, 2004; Rehbein et al., 2010; Choo et al., 2010; Gentile et al., 2011). So gaben beispielsweise in einer Befragung von Onlinerollenspielern von Griffiths und Kollegen (Griffiths et al., 2004) 13 Prozent der Jugendlichen und 21 Prozent der Erwachsenen an, aufgrund des Spielens weniger zu schlafen. In der Befragung von Rehbein und Kollegen (Rehbein et al., 2010) von 15.168 im Mittel 15-jährigen Jugendlichen berichteten insbesondere Jugendliche mit einem problematischen Computerspielverhalten kürzere Schlafenszeiten sowie erhöhte Schlafprobleme. Ferner zeigen sich bei Mustern exzessiver Computerspielnutzung auch Anzeichen für Schlafentzug, welcher mit einem problematischerem Sozialverhalten, verringerter Konzentrationsfähigkeit und verminderter psychischer Gesundheit in Zusammenhang stehen könnte (vgl. Gillespie, 2002; Tazawa \& Okada, 2001).

Insgesamt muss wohl von einem komplexen Zusammenspiel sowie von einer gegenseitigen Beeinflussung der unterschiedlichen Wirkpfade (Bewegungsmangel/Ernährung/Schlaf) ausgegangen werden, wobei sich diese zudem vor dem Hintergrund bestehender protektiver oder Risikofaktoren (vgl. Abbildung 78) interindividuell auch unterschiedlich auswirken dürften.

\subsection{Ergebnisse des Berliner Längsschnitt Medien - Mediennutzung und Fettleibigkeit}

Ein weiterer Themenschwerpunkt des Berliner Längsschnitts Medien war die Beantwortung der Frage, in welcher Weise sich zeitlich exzessive Mediennutzung auf Kinder bezüglich ihrer körperlichen Entwicklung auswirkt. So zeigen epidemiologische Untersuchungen im Allgemeinen zunächst eine zunehmende Bewegungsarmut bei Kindern und Jugendlichen und eine Abnahme der körperlichen Fitness (z. B. Schmidt, W. et al., 2003). Zum Teil ist zudem eine Verdrän-

207 Die Autoren selbst betonen jedoch den explorativen Charakter dieser Studie, da zum einen nur Haupt- und Realschüler befragt wurden und zum anderen weder die ethnische Zugehörigkeit noch das Bildungsniveau als wichtige Einflussfaktoren auf die Medienzeiten berücksichtigt wurden. 
gung der Bewegungsaktivitäten durch eine intensive Mediennutzung von Kindern und Jugendlichen zu beobachten (vgl. Marshall et al., 2004), wodurch langfristig mit Übergewicht und Fettleibigkeit bei exzessiven Mediennutzern gerechnet werden muss (vgl. Stettler et al., 2004). Erhöhte sportliche Aktivität wirkt sich auf der anderen Seite positiv auf die soziale Entwicklung, die sozialen Kontakte, die Konzentrationsfähigkeit sowie die Lernfreude der Kinder aus (Müller, C. \& Petzold, 2002; Myrtek \& Scharff, 2000). Neurobiologischer Forschung zufolge steht Bewegung mit Intelligenzentwicklung in direktem Zusammenhang. So spielt Sport eine wichtige Rolle für die Hirndurchblutung und die Vernetzung der Hirnzellen untereinander (Kubesch, 2002, 2004).

Von maßgeblicher Bedeutung für den Berliner Längsschnitt Medien sind zwei Fragen: Lassen sich erstens die Zusammenhänge zwischen einer zeitlich exzessiven Mediennutzung und einer erhöhten Fettleibigkeit bestätigen und zwar bei Berücksichtigung der Bedingungsfaktoren Wohlstand und Bildungshintergrund im Elternhaus, welche sich unmittelbar auf die Mediennutzungsgewohnheiten der Kinder auswirken (Kapitel 6.2.2)? Können diese Zusammenhänge auch hinsichtlich einer Wirkungsvermutung im Gegensatz zu einer Selektion im Längsschnitt bestätigt werden (Kapitel 6.2.3)?

\subsubsection{Entwicklung von Körpergröße, Körpergewicht und BMI im Grundschulalter}

Von der ersten Befragungswelle an wurden an einem der beiden Untersuchungstage das Körpergewicht (in Hose und T-Shirt) ${ }^{208}$ sowie die Körpergröße der Kinder mit geeichten Messinstrumenten ${ }^{209}$ zur Berechnung des Body-Mass-Index ${ }^{210}$ (BMI) bestimmt. Die Kinder wurden anschließend hinsichtlich Körpergröße, Körpergewicht und BMI nach einer Einteilung von Zwiauer und Wabitsch (1997) -halbjahresgenauer geschlechtsspezifischer Altersnormen für das Alter von 7 bis 14 Jahren - in unterschiedliche Größen-, Gewichts- und BMI-Gruppen eingeteilt.

Körpergröße. Von Beginn der Längsschnittstudie im November 2005 an war die Stichprobe der Berliner Grundschüler- und Grundschülerinnen mit im Durch-

208 Da die Messung in T-Shirt und Hose zu einer überproportionalen Anzahl von übergewichtigen Kindern führen würde, wurde, um das zusätzliche Gewicht von T-Shirt und Hose zu berücksichtigen, zu jedem Messzeitpunkt ein Textilfaktor berechnet der vor der Berechnung des Body-Mass-Index vom Körpergewicht der Kinder subtrahiert wurde. Der Textilfaktor berechnet sich nach folgender Formel:

209 Waage seca 862, Längenmessstab seca 214.

textilfaktor $=\sqrt{\frac{\text { Körpergewicht } \times \text { Körpergröße }}{\text { Stichprobenmittelwert }(\text { Körpergewicht } \times \text { Körpergröße })}}$.

210 Körpergewicht in Kilogramm/(Körpergröße in Metern $)^{2}$. 
schnitt $1.38 \mathrm{~m}$ insgesamt etwas größer als die Kinder der Normstichprobe (vgl. Tabelle 77). So wurden auch aufgrund der Messergebnisse des zweiten Befragungszeitpunktes am Ende der dritten Klasse ${ }^{211}$ lediglich 7.9 Prozent der Jungen und 6.6 Prozent der Mädchen unter die unteren zehn Prozent $(\mathrm{P} \leq 10)$, aber 9.7 Prozent der Jungen und 13.5 Prozent der Mädchen unter die oberen zehn Prozent der Normstichprobe $(\mathrm{P}>90)$ eingestuft. Bis zum Ende der sechsten Klasse vergrößerten sich die Unterschiede zwischen diesen beiden Extrembereichen der Verteilung zudem und zwar etwas deutlicher bei den Jungen: Beim letzten Messzeitpunkt fielen einerseits nur noch 5.4 Prozent der Jungen und 5.6 Prozent der Mädchen unter die unteren zehn Prozent $(\mathrm{P} \leq 10)$. Andererseits wurden 17.3 Prozent der Jungen und 13.1 Prozent der Mädchen unter die oberen zehn Prozent der Normstichprobe $(\mathrm{P}>90)$ eingeordnet.

Körpergewicht. Ein vergleichbarer Trend ist auch für die Daten zum Körpergewicht festzustellen, hier allerdings mit deutlicheren Unterschieden zwischen den Messzeitpunkten bei den Mädchen. Am Ende der dritten Klasse wurden lediglich 9.1 Prozent der Jungen und 9.3 Prozent der Mädchen unter die unteren zehn Prozent $(\mathrm{P} \leq 10)$, aber bereits 15 Prozent der Jungen und 14.8 Prozent der Mädchen unter die oberen zehn Prozent der Normstichprobe $(\mathrm{P}>90)$ eingestuft. Bis zum letzten Messzeitpunkt vergrößerten sich diese Unterschiede merklich für beide Geschlechter, mit einer ausgeprägten Zunahme am oberen Ende der Verteilung: Am Ende der sechsten Klasse fielen auf der einen Seite nur noch 5.7 Prozent der Jungen und 7.1 Prozent der Mädchen unter die unteren zehn Prozent $(\mathrm{P} \leq 10)$. Auf der anderen Seite wurden 21.6 Prozent der Jungen und 21.8 Prozent der Mädchen aufgrund ihres Körpergewichts unter die oberen zehn Prozent der Normstichprobe $(\mathrm{P}>90)$ eingeordnet.

211 Die Messdaten zum ersten Messzeitpunkt im November 2005 werden hier aufgrund der fehlenden Vergleichbarkeit insbesondere im Zusammenhang mit den Daten der Mediennutzung nicht dargestellt. 
Tabelle 77. Körpergröße, Körpergewicht und BMI nach Geschlecht und Jahrgangsstufe.

\begin{tabular}{|c|c|c|c|c|c|c|c|c|}
\hline \multirow[b]{2}{*}{ Klasse } & \multicolumn{4}{|c|}{ Jungen } & \multicolumn{4}{|c|}{ Mädchen } \\
\hline & 3. & 4. & 5. & 6. & 3. & 4. & 5. & 6. \\
\hline Körpergröße & $1.38 \mathrm{~m}$ & $1.43 \mathrm{~m}$ & $1.49 \mathrm{~m}$ & $1.56 \mathrm{~m}$ & $1.38 \mathrm{~m}$ & $1.44 \mathrm{~m}$ & $1.51 \mathrm{~m}$ & $1.57 \mathrm{~m}$ \\
\hline$\leq \mathrm{P} 3$ & $1.0 \%$ & $2.4 \%$ & $1.2 \%$ & $0.7 \%$ & $1.8 \%$ & $1.8 \%$ & $2.3 \%$ & $2.8 \%$ \\
\hline$\leq \mathrm{P} 10$ & $6.9 \%$ & $5.6 \%$ & $3.7 \%$ & $4.7 \%$ & $4.8 \%$ & $2.5 \%$ & $2.5 \%$ & $2.8 \%$ \\
\hline$\leq \mathrm{P} 50$ & $40.7 \%$ & $40.6 \%$ & $35.5 \%$ & $31.4 \%$ & $40.6 \%$ & $37.8 \%$ & $34.5 \%$ & $32.4 \%$ \\
\hline$\leq \mathrm{P} 90$ & $41.7 \%$ & $39.5 \%$ & $44.9 \%$ & $45.8 \%$ & $39.3 \%$ & $43.4 \%$ & $46.6 \%$ & $49.5 \%$ \\
\hline$\leq \mathrm{P} 97$ & $6.7 \%$ & $7.5 \%$ & $10.9 \%$ & $11.6 \%$ & $8.0 \%$ & $8.9 \%$ & $8.6 \%$ & $7.3 \%$ \\
\hline > P 97 & $3.0 \%$ & $4.3 \%$ & $3.7 \%$ & $5.7 \%$ & $5.5 \%$ & $5.6 \%$ & $5.5 \%$ & $5.8 \%$ \\
\hline Körpergewicht & $\begin{array}{c}33.4 \\
\mathrm{~kg}\end{array}$ & $\begin{array}{c}37.3 \\
\mathrm{~kg}\end{array}$ & $\begin{array}{c}42.0 \\
\mathrm{~kg}\end{array}$ & $\begin{array}{c}48.1 \\
\mathrm{~kg}\end{array}$ & $\begin{array}{c}33.2 \\
\mathrm{~kg}\end{array}$ & $\begin{array}{c}37.6 \\
\mathrm{~kg}\end{array}$ & $\begin{array}{c}43.9 \\
\mathrm{~kg}\end{array}$ & $49.5 \mathrm{~kg}$ \\
\hline$\leq \mathrm{P} 3$ & $3.2 \%$ & $2.4 \%$ & $1.5 \%$ & $1 \%$ & $3.0 \%$ & $1.8 \%$ & $1.8 \%$ & $1.8 \%$ \\
\hline$\leq \mathrm{P} 10$ & $5.9 \%$ & $5.3 \%$ & $5.0 \%$ & $4.7 \%$ & $6.3 \%$ & $6.4 \%$ & $5.3 \%$ & $5.3 \%$ \\
\hline$\leq \mathrm{P} 50$ & $40.3 \%$ & $37.9 \%$ & $37.8 \%$ & $34.2 \%$ & $36.5 \%$ & $33.2 \%$ & $31.6 \%$ & $27.1 \%$ \\
\hline$\leq \mathrm{P} 90$ & $35.6 \%$ & $35.9 \%$ & $37.3 \%$ & $38.5 \%$ & $39.3 \%$ & $41.8 \%$ & $39.7 \%$ & $44.1 \%$ \\
\hline$\leq \mathrm{P} 97$ & $11.1 \%$ & $13.3 \%$ & $12.3 \%$ & $15.1 \%$ & $10.3 \%$ & $11.2 \%$ & $13.7 \%$ & $13.2 \%$ \\
\hline > P 97 & $3.9 \%$ & $5.1 \%$ & $6.0 \%$ & $6.5 \%$ & $4.5 \%$ & $5.6 \%$ & $7.9 \%$ & $8.6 \%$ \\
\hline BMI & 17.3 & 18.0 & 18.7 & 19.6 & 17.4 & 18.0 & 19.2 & 20.0 \\
\hline $\begin{array}{l}\text { ausgeprägtes } \\
\text { Untergewicht }\end{array}$ & $1.5 \%$ & $1.9 \%$ & 2.3 & $1.2 \%$ & 2. & $\%$ & $3.1 \%$ & $2.5 \%$ \\
\hline Untergewicht & $8.6 \%$ & $8.0 \%$ & $5.8 \%$ & $6.5 \%$ & $7.8 \%$ & $6.6 \%$ & $6.1 \%$ & $5.6 \%$ \\
\hline $\begin{array}{l}\text { Normalge- } \\
\text { wicht }\end{array}$ & $73.5 \%$ & $70.4 \%$ & $72.0 \%$ & $72.5 \%$ & $73.6 \%$ & $74.5 \%$ & $71.2 \%$ & $71.1 \%$ \\
\hline Übergewicht & $12.3 \%$ & $14.3 \%$ & $14.9 \%$ & $12.7 \%$ & $12.6 \%$ & $12.0 \%$ & $12.5 \%$ & $13.4 \%$ \\
\hline Adipositas & $4.2 \%$ & $5.3 \%$ & $5.0 \%$ & $7.2 \%$ & $3.5 \%$ & $4.1 \%$ & $7.1 \%$ & $7.3 \%$ \\
\hline
\end{tabular}

BMI. Analog zu den Ergebnissen zum Körpergewicht sowie zur Körpergröße, sind auch hinsichtlich des BMI der Kinder, der diese beiden Größen zueinander in Beziehung setzt, zwischen den Messzeitpunkten für beide Geschlechter vor allem Veränderungen am oberen Extrem der Verteilung, d. h. unter den übergewichtigen und adipösen Kindern, zu beobachten. Am Ende der dritten Klasse im Mai 2006 wurden aufgrund der Daten zum BMI 10.1 Prozent der Jungen und 10.3 Prozent der Mädchen der Berliner Stichprobe als untergewichtig sowie bereits 16.5 Prozent der Jungen und 16.1 Prozent der Mädchen als übergewichtig oder adipös klassifiziert. Bis zum Ende der sechsten Klasse änderte sich der Anteil der untergewichtigen Kinder nur unmerklich; bei den Jungen auf 7.7 Prozent sowie bei den Mädchen auf 8.1 Prozent. Der Anteil der adipösen und überge- 
wichtigen Schülerinnen und Schüler stieg dagegen auf 19.9 Prozent bei den Jungen sowie 20.7 Prozent bei den Mädchen an. Auch wenn ein geringer Anteil dieser als übergewichtig oder adipös eingestuften Kinder möglicherweise aufgrund von Messungenauigkeiten (Messung in T-Shirt und Hose, Textilfaktor) falsch klassifiziert wurde, ist dennoch für die vorliegende Stichprobe der Berliner Grundschülerinnen und Grundschüler ein substanzieller Prozentsatz von Kindern mit einem im klinischen Sinne auffälligen bis sehr auffälligen BMI zu konstatieren.

Bevor das Risiko einer erhöhten Fettleibigkeit mit einer zeitlich exzessiven Mediennutzung in Beziehung gesetzt wird, sollen zunächst Zusammenhänge zwischen dem BMI der Kinder und den sozial-strukturellen Bedingungsfaktoren Migrationsstatus, Wohlstand der Familie sowie Bildungshintergrund im Elternhaus dargestellt werden, die sich allesamt auch unmittelbar auf die Mediennutzungsgewohnheiten der Kinder auswirken (vgl. Kapitel 2). Aufgeführt in Tabelle 78 sind wiederum aus Gründen der Vergleichbarkeit jeweils nur der BMI gemessen am Ende der dritten, vierten, fünften und sechsten Klasse.

Tabelle 78. Zusammenhänge BMI und sozial-strukturelle Merkmale.

\begin{tabular}{|c|c|c|c|c|}
\hline & \multicolumn{4}{|c|}{$\overline{\text { BMI }}$} \\
\hline & $\begin{array}{l}\text { 3. Klasse } \\
M \text { (SD) }\end{array}$ & $\begin{array}{l}\text { 4. Klasse } \\
M(S D)\end{array}$ & $\begin{array}{l}\text { 5. Klasse } \\
M(S D)\end{array}$ & $\begin{array}{l}\text { 6. Klasse } \\
M(S D)\end{array}$ \\
\hline Gesamt & $17.32(2.83)$ & $18.02(3.19)$ & $18.95(3.69)$ & $19.78(3.78)$ \\
\hline \multicolumn{5}{|c|}{ Migrationshintergrund } \\
\hline Deutsch & $17.17(2.71)$ & $17.86(3.11)$ & $18.65(3.58)$ & $19.59(3.76)$ \\
\hline MH & $17.77(3.11)^{*}$ & $18.49(3.35)^{*}$ & $19.71(3.88)^{* *}$ & $20.25(3.80)^{*}$ \\
\hline \multicolumn{5}{|c|}{ Bildungsniveau } \\
\hline niedrig & $17.61(3.34)$ & $18.38(3.66)$ & $19.31(3.87)$ & $20.13(4.14)$ \\
\hline mittel & $17.55(2.96)$ & $18.36(3.36)$ & $19.26(3.75)$ & $20.31(4.04)$ \\
\hline hoch & $17.10(2.51)^{\ddot{I}}$ & $17.67(2.85)^{*}$ & $18.47(3.33)^{* *}$ & $19.19(3.31)^{*}$ \\
\hline \multicolumn{5}{|l|}{ Wohlstand } \\
\hline Q1 & $17.27(2.66)$ & $18.33(3.68)$ & $19.44(4.38)$ & $20.14(4.04)$ \\
\hline Q2 & $17.54(2.99)$ & $18.45(3.28)$ & $19.39(3.79)$ & $20.27(3.97)$ \\
\hline Q3 & $17.61(3.07)$ & $18.16(3.26)$ & $19.09(3.97)$ & $20.00(4.25)$ \\
\hline Q4 & $17.23(2.41)$ & $17.88(2.67)$ & $18.71(2.89)$ & $19.62(3.35)$ \\
\hline Q5 & $16.80(2.49)^{\ddot{I}}$ & $17.25(2.75)^{* *}$ & $18.01(3.09)^{* *}$ & $18.75(2.86)^{* *}$ \\
\hline
\end{tabular}

Anmerkung. Signifikanzen für nicht gleiche Varianzen berichtet für: Migrationshintergrund. Für Migrationshintergrund wurde ein t-Tests für unabhängige Stichproben, für alle anderen Variablen eine ANOVA berechnet. $* p<.05, * * p<.01, \mathrm{I} p<.10$.

Insgesamt ist erwartungsgemäß eine Zunahme im BMI der Schülerinnen und Schüler von der dritten Klasse bis zur sechsten Klasse zu beobachten, die bei Be- 
rechnung der geschlechtsspezifischen Altersnormen allerdings berücksichtigt wird (vgl. Zwiauer \& Wabitsch, 1997). Bezüglich des BMI ist hinsichtlich des Migrationshintergrundes der Kinder am Ende der dritten Klasse ein kleiner Vorsprung der Kinder mit gegenüber den Kindern ohne Migrationshintergrund dahin gehend zu beobachten, dass Kinder aus Familien mit Migrationshintergrund im Mittel einen etwas höheren BMI haben. Dieser Unterschied bleibt bis zur sechsten Klasse bei einer insgesamt größeren Spannbreite stabil. Betrachtet man das Bildungsniveau im Elternhaus, sind vergleichbare Unterschiede im BMI festzustellen, wobei sich vor allem Kinder aus Familien mit hohem Bildungsniveau von den anderen beiden Gruppen unterscheiden. Kinder aus Familien mit niedrigem Bildungsniveau (höchster Bildungsabschluss beider Elternteile ist der Hauptschulabschluss) und Kinder aus Familien mit mittlerem Bildungsniveau (höchster Bildungsabschluss mindestens eines Elternteils ist die Mittlere Reife) haben zu allen Messzeitpunkten einen vergleichbaren mittleren BMI. Kinder aus Familien mit hohem Bildungsniveau (höchster Bildungsabschluss mindestens eines Elternteils ist ein abgeschlossenes Studium oder das Abitur) sind hingegen zu allen Messzeitpunkten deutlich weniger übergewichtig oder adipös. Dieser Unterschied vergrößert sich zudem von der dritten bis zur sechsten Klasse. Die Befunde zum Zusammenhang von familiärem Wohlstand und BMI sind annähernd vergleichbar mit denen zum Bildungsniveau im Elternhaus. $\mathrm{Zu}$ allen drei Messzeitpunkten ist mit steigendem familiärem Wohlstand ein geringer BMI festzustellen. Analog zur Unterscheidung nach Bildungsniveau im Elternhaus verfestigen sich diese Unterschiede ebenfalls von der dritten bis zur sechsten Klasse.

\subsubsection{Korrelative Zusammenhänge zwischen Mediennutzung und Fettleibigkeit}

Um wiederum einen ersten Eindruck zur unterschiedlichen Bedeutung einer zeitlich exzessiven Computerspiel- und Fernsehnutzung im Zusammenhang mit dem erhöhten Risiko einer Fettleibigkeit zu erhalten, wurden zunächst quer- sowie längsschnittliche Partialkorrelationen zwischen dem BMI und den unterschiedlichen Medienvariablen ${ }^{212}$ berechnet. Kontrolliert wurde dabei für den Einfluss des familiären Wohlstands und des Bildungsniveaus im Elternhaus (vgl. Tabelle 79 und Tabelle 79).

212 Fernsehzeit und Computerspielzeit wurden mit der Schätzfrage im umcodierten Einstundenabstand erhoben. TV läuft während des Essens wurde in der dritten Klasse mit der Frage „Wie oft läuft der Fernseher bei euch zu Hause während des Abendbrots?“ in der fünften und sechsten Klasse mit der Frage „Wie oft läuft der Fernseher bei euch zu Hause während des Essens?" mit den Abstufungen „das ist immer so“, „das ist meistens so“, „das ist manchmal so“, „das ist nie so“, erhoben. 
Tabelle 79. Partialkorrelationen Mediennutzungszeiten (Fernsehen) und BMI.

\begin{tabular}{|c|c|c|c|c|c|c|c|c|c|c|c|}
\hline \multirow[b]{2}{*}{ Klasse } & \multicolumn{4}{|c|}{ Schultag } & \multicolumn{4}{|c|}{ Samstag } & \multicolumn{3}{|c|}{$\begin{array}{l}\text { TV läuft während des } \\
\text { Essens }\end{array}$} \\
\hline & 3. & 4. & 5. & 6. & 3. & 4. & 5. & 6. & 3. & 5. & 6. \\
\hline \multicolumn{12}{|l|}{ BMI } \\
\hline 3. Klasse & $.06^{\mathrm{ns}}$ & $.15^{* *}$ & $.13^{* *}$ & $.14^{* *}$ & $.10^{*}$ & $.14 * *$ & $.06^{\mathrm{ns}}$ & $.02^{\mathrm{ns}}$ & $.10^{*}$ & $.07^{\mathrm{ns}}$ & $.02^{\mathrm{ns}}$ \\
\hline 4. Klasse & $.04^{\mathrm{ns}}$ & $.16^{* *}$ & $.10^{\mathrm{i}}$ & $.10^{*}$ & $.08^{\mathrm{ns}}$ & $.11^{*}$ & $.07^{\mathrm{ns}}$ & $-.02^{\mathrm{ns}}$ & $.11^{* *}$ & $.05^{\mathrm{ns}}$ & $.00^{\mathrm{ns}}$ \\
\hline 5. Klasse & $.07^{\mathrm{ns}}$ & $.17^{* *}$ & $.14^{* *}$ & $.15^{* *}$ & $.09^{\hat{\imath}}$ & $.15^{* *}$ & $.08^{\mathrm{ns}}$ & $.02^{\mathrm{ns}}$ & $.09^{\hat{\imath}}$ & $.08^{\hat{\imath}}$ & $.05^{\mathrm{ns}}$ \\
\hline 6. Klasse & $.05^{\mathrm{ns}}$ & $.16^{* *}$ & $.11^{*}$ & $.14^{* *}$ & $.10^{\hat{\imath}}$ & $.11^{*}$ & $.06^{\mathrm{ns}}$ & $.03^{\hat{\imath}}$ & $.11^{*}$ & $.06^{\mathrm{ns}}$ & $.04^{\mathrm{ns}}$ \\
\hline
\end{tabular}

Anmerkung. Kontrollierte Variablen: Familiärer Wohlstand und Bildungsniveau im Elternhaus. * $p$ $<.05, * * p<.01$, i $p<.10$.

Tabelle 80. Partialkorrelationen Mediennutzungszeiten (Computerspiele) und BMI.

\begin{tabular}{|c|c|c|c|c|c|c|c|c|}
\hline \multirow[b]{2}{*}{ Klasse } & \multicolumn{4}{|c|}{ Schultag } & \multicolumn{4}{|c|}{ Samstag } \\
\hline & 3. & $\overline{4 .}$ & 5. & $\overline{6 .}$ & 3. & 4. & 5. & 6. \\
\hline \multicolumn{9}{|l|}{ BMI } \\
\hline 3. Klasse & $.08^{\text {ns }}$ & $.11 *$ & $.06^{\mathrm{ns}}$ & $.07^{\mathrm{ns}}$ & $.14 * *$ & $.11^{*}$ & $.15^{* *}$ & $.01^{\mathrm{ns}}$ \\
\hline 4. Klasse & $.07^{\mathrm{ns}}$ & $.12 *$ & $.06^{\mathrm{ns}}$ & $.04^{\mathrm{ns}}$ & $.13^{* *}$ & $.10^{*}$ & $.14 * *$ & $.01^{\mathrm{ns}}$ \\
\hline 5. Klasse & $.08^{\mathrm{ns}}$ & $.12 *$ & $.08^{\mathrm{ns}}$ & $.07^{\mathrm{ns}}$ & $.13^{*}$ & $.11^{*}$ & $.15^{* *}$ & $.02^{\mathrm{ns}}$ \\
\hline 6. Klasse & $.09^{\hat{\imath}}$ & $.10^{\hat{\imath}}$ & $.05^{\mathrm{ns}}$ & $.07^{\mathrm{ns}}$ & $.14^{* *}$ & $.10^{\hat{\imath}}$ & $.14 * *$ & $.00^{\mathrm{ns}}$ \\
\hline
\end{tabular}

Anmerkung. Kontrollierte Variablen: Familiärer Wohlstand und Bildungsniveau im Elternhaus. ${ }^{*} p$ $<.05, * * p<.01, \dddot{\imath} p<.10$.

Bezüglich der täglichen Mediennutzungszeiten an einem Schultag zeigen sich, bei Kontrolle des familiären Wohlstands sowie des Bildungsniveaus im Elternhaus, vor allem in der vierten, fünften und sechsten Klasse und vor allem für das Fernsehen statistisch bedeutsame geringe positive Zusammenhänge mit dem BMI der Kinder. Betrachtet man hingegen die täglichen Mediennutzungszeiten an einem Samstag, kann vor allem ein Zusammenhang zwischen höheren Computerspielzeiten und einer erhöhten Fettleibigkeit beobachtet werden. In der längsschnittlichen Betrachtung legen die Korrelationen zwischen den beiden Medien und dem BMI der Schülerinnen und Schüler vor allem eine Wechselwirkung nahe, d. h., unabhängig vom familiären Wohlstand und dem Bildungsniveau im Elternhaus spielen übergewichtige und adipöse Kinder länger Computerspiele und schauen länger Fernsehen, was wiederum das Risiko einer Fettleibigkeit erhöht. Den stärksten Einfluss entwickeln dabei die Fernseh- und Computerspielnutzungszeiten der vierten bzw. fünften Klasse. Dasselbe Bild ergibt sich auch bei Betrachtung der Frage, ob der Fernseher beim Essen läuft. Unab- 
hängig vom familiären Wohlstand und dem Bildungsniveau im Elternhaus haben Kinder, bei denen der Fernseher während des Essens läuft, sowohl in der dritten als auch in der fünften Klasse im Mittel einen höheren BMI.

\subsubsection{Längsschnittlicher Zusammenhang zwischen Mediennutzung und Fettleibigkeit}

Zur genaueren längsschnittlichen Überprüfung des Wirkzusammenhangs exzessiv zeitlicher Mediennutzung und einer erhöhten Fettleibigkeit der Kinder, wurde ein weiteres Strukturgleichungsmodell für die vier hinsichtlich des Befragungstermins vergleichbaren Messzeitpunkte (Ende der 3., 4., 5. und 6. Klasse) mit dem fünffach abgestuften BMI der Kinder (,,ausgeprägtes Untergewicht“, „Untergewicht", „Normalgewicht“, „Übergewicht“, „Adipositas") berechnet (siehe Abbildung 79). Zur gleichzeitigen Berücksichtigung der Fernseh- und Computerspielzeiten an einem Schul- sowie an einem Samstag wurde ein Index zeitlicher Mediennutzung berechnet. Hierfür wurden die beiden Indizes Mediennutzung $\mathrm{tv}_{\mathrm{v}}$ und Mediennutzung $\mathrm{PC}_{\mathrm{PC}}$ aufsummiert (vgl. Kapitel 2.2.5). Aufgenommen in die Analyse wurden dabei nur Personen, zu denen zum zweiten, dritten, vierten oder fünften Messzeitpunkt und insgesamt mindestens zu zwei Messzeitpunkten Daten vorlagen. Aufgrund unterschiedlicher Mediennutzungsgewohnheiten von Jungen und Mädchen wurde das Geschlecht der Kinder wiederum insofern berücksichtigt, als dass zusätzlich getrennte Modelle jeweils für Jungen und Mädchen berechnet wurden.

Das Modell213 (siehe Abbildung 79) zeigt zunächst bei guter Modellpassung (Gesamt: $\chi 2=15.90(9, N=897), R M S E A=.03, T L I=.99, C F I=.99$; Jungen: $\chi^{2}=10.79(9, N=456), R M S E A=.02, T L I=.99, C F I=.99$; Mädchen: $\chi 2=13.81$ $(9, N=441), R M S E A=.04, T L I=.99, C F I=.99)$ für die dritte Klasse einen geringen, querschnittlichen korrelativen Zusammenhang $(\varphi=.06)$ zwischen höheren Mediennutzungszeiten und einem erhöhten BMI der Kinder. Dieser Zusammenhang fällt jedoch für die Jungen $\left(\varphi=.04^{\text {ns }}\right)$ deutlich geringer als für die Mädchen $(\varphi=.10)$ aus und ist auch nur für letztere statistische bedeutsam. Über die Messzeitpunkte betrachtet, ist der BMI (Gesamt: $\beta_{34}=.92, \beta_{45}=.65, \beta_{56}=.52$, Jungen: $\beta_{34}=.92, \beta_{45}=.56, \beta_{56}=.49$, Mädchen: $\left.\beta_{34}=.93, \beta_{45}=.74, \beta_{56}=.55\right)$ das Merkmal, das weniger Veränderung unterliegt (Mediennutzungszeiten; Gesamt: $\beta_{34}=.57$, $\beta_{45}=.42, \beta_{56}=.50$, Jungen: $\beta_{34}=.67, \beta_{45}=.68, \beta_{56}=.77$, Mädchen: $\beta_{34}=.79, \beta_{45}=$.

213 Als Stichprobenauswahl wurden nur Kinder der Interventions- und Kontrollklassen aufgenommen, zu denen zum zweiten, dritten, vierten oder fünften Messzeitpunkt und insgesamt mindestens zu zwei Messzeitpunkten Daten vorlagen. 
$71, \beta_{56}=.71$ ), wobei die Stabilitäten für BMI und Mediennutzungszeiten über die beiden Geschlechter vergleichbar sind.

Im Gesamtmodell deuten die Kreuzpfade zwischen den Messzeitpunkten bei einer insgesamt geringen Höhe (was sich in den Partialkorrelationen bereits angedeutet hatte) auf eine einseitige Beeinflussung in dem Sinne hin, dass Kinder mit einem hohen BMI verstärkt digitale Bildschirmmedien konsumieren. Bei einer nach Geschlecht getrennten Betrachtung bestätigt sich dieses Modell für die Mädchen, nicht jedoch für die Jungen. So legt das Modell der Mädchen, wie bereits für den Zusammenhang von Mediennutzungszeiten (Fernsehen bzw. Computerspiele) und Schulnoten berichtet, wiederum nahe, dass Mädchen mit einem hohen BMI verstärkt Fernsehprogramme schauen und Computerspiele spielen (ohne eine Wirkung in die andere Richtung). Für die Jungen könnte auf der anderen Seite, bei insgesamt geringeren (und fast durchwegs nicht statistisch bedeutsamen) Pfadkoeffizienten noch eher eine wechselnde Beeinflussung in zeitlicher Abfolge, vergleichbar mit den Befunden zur Schulleistung, angenommen werden. 


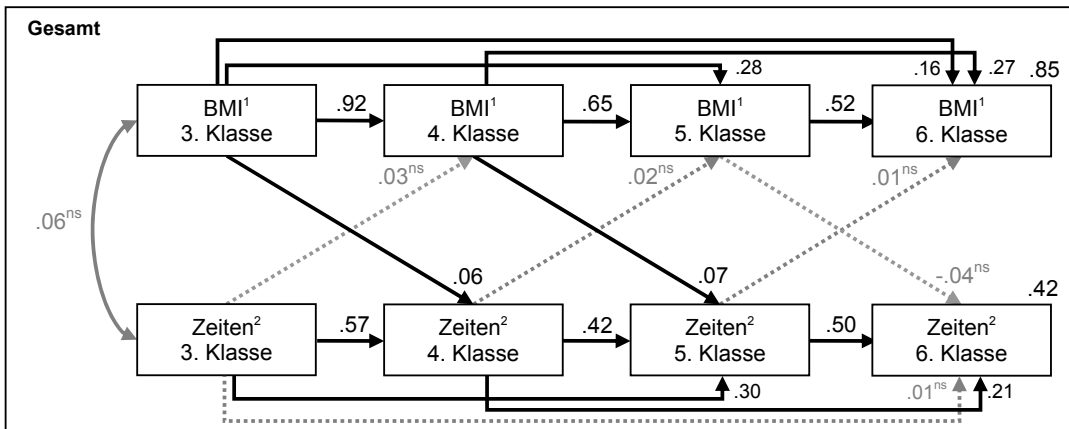

Modell Fit:

$\mathrm{X}^{2}=15.90 \mathrm{df}=9, \mathrm{TLI}=.99, \mathrm{CFI}=.99, \mathrm{RMSEA}=.03$, Standardisierte Parameter; alle Pfade mindestens $p \leq .05$ (falls nicht anders gekennzeichnet).

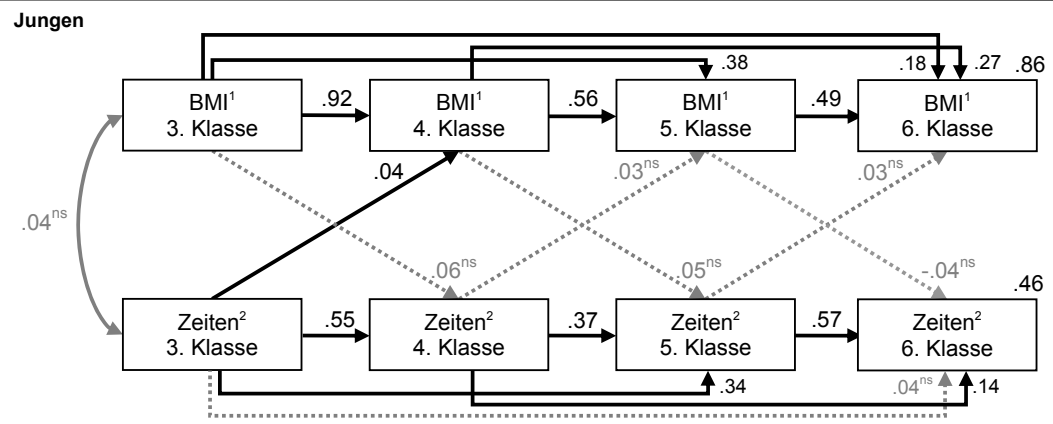

Modell Fit:

$\mathrm{X}^{2}=10.79 \mathrm{df}=9, \mathrm{TLI}=.99, \mathrm{CFI}=.99, \mathrm{RMSEA}=.02$, Standardisierte Parameter; alle Pfade mindestens $p \leq .05$ (falls nicht anders gekennzeichnet).

\section{Mädchen}

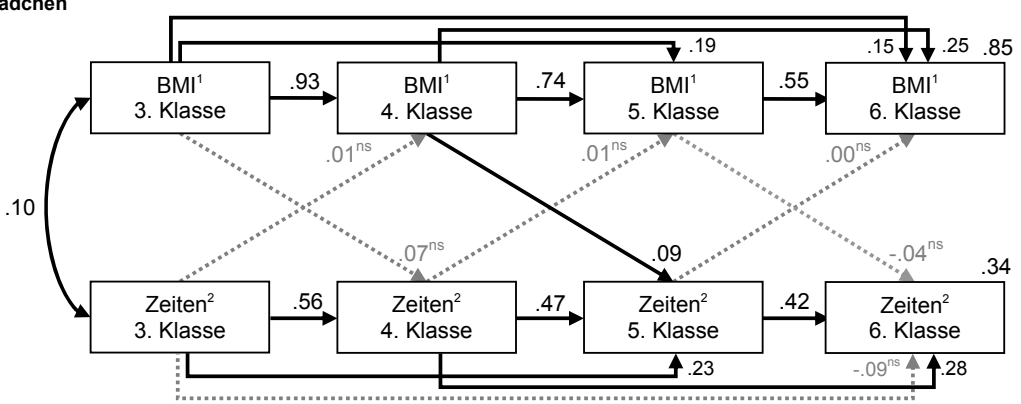

Modell Fit:

$X^{2}=13.81 \mathrm{df}=9, \mathrm{TLI}=.99, \mathrm{CFI}=.99, \mathrm{RMSEA}=.04$, Standardisierte Parameter; alle Pfade mindestens $p \leq .05$ (falls nicht anders gekennzeichnet).

${ }^{1}$ Body Mass Index (kategorial): ausgeprägtes Untergewicht, Untergewicht, Normalgewicht, Übergewicht, Adipositas

${ }^{2}$ Index wöchentlicher Mediennutzung: Summe Index wöchentlicher Mediennutzung ${ }_{\mathrm{TV}}$, Index wöchentlicher

Mediennutzung ${ }_{\mathrm{PC}}$

Abbildung 79. Strukturgleichungsmodelle zum längsschnittlichen Zusammenhang von Mediennutzungszeiten und Fettleibigkeit. 


\subsection{Diskussion}

In Kapitel 6.2 wurden zum Thema häusliche Mediennutzung und Fettleibigkeit die folgenden beiden Leitfragen formuliert, die mit dem Berliner Längsschnitt Medien beantwortet werden sollten:

1. Lassen sich die Zusammenhänge zwischen einer zeitlich exzessiven Mediennutzung und einer erhöhten Fettleibigkeit bei Berücksichtigung der Bedingungsfaktoren Wohlstand und Bildungshintergrund im Elternhaus, welche sich unmittelbar auf die Mediennutzungsgewohnheiten der Kinder auswirken, bestätigen?

2. Können Befunde hinsichtlich einer Wirkungsvermutung im Sinne von Ursächlichkeit (Hohe Mediennutzungszeiten führen zu Fettleibigkeit) im Gegensatz zu einer Selektion (fettleibigere Kinder nutzen eher länger Medien) im Längsschnitt bestätigt werden?

Im Folgenden sollen nach einer kurzen Betrachtung der Ergebnisse zur Entwicklung des BMI die beiden Fragenkomplexe einzeln aufgegriffen und mit der bestehenden Forschungslage in Beziehung gesetzt werden.

Hinsichtlich Übergewicht und Adipositas konnten im Berliner Längsschnitt Medien mit dem für Deutschland repräsentativen Kinder- und Jugendgesundheitssurvey (KiGGS) des Robert-Koch-Institutes vergleichbare Prävalenzwerte ermittelt werden: In der Befragung am Ende der dritten Klasse wurden 16.5 Prozent der Schüler bzw. 16.1 Prozent der Schülerinnen des Berliner Längsschnitt Medien als übergewichtig oder adipös eingestuft. In der vergleichbaren Altersgruppe der 7-10-jährigen Kinder des KiGGS waren dies 15.9 Prozent der Schüler respektive 14.7 Prozent der Schülerinnen (Kurth, B. \& Schaffrath, 2007). Auffällig ist jedoch, dass bei einer separaten Betrachtung der Kategorien übergewichtig und adipös im Berliner Längsschnitt Medien eine leichte Verschiebung zu Gunsten des Übergewichts zu beobachten ist (Übergewicht: BLM - Jungen: $12.3 \%$, KiGGS - Jungen: $8.9 \%$; $B L M$ - Mädchen: $12.6 \%$, KiGGS - Mädchen: $9.0 \%$; Adipositas: $B L M$ - Jungen: $4.2 \%$, KiGGS - Jungen: 7.0\%; BLM - Mädchen: $3.5 \%$, KiGGS - Mädchen: $5.7 \%$ ). Die leichten Abweichungen in der Gesamtprävalenz sowie die Verschiebungen können dabei durch das ältere Durchschnittsalter der Schülerinnen und Schüler des Berliner Längsschnitt Medien in der dritten Klasse (9;6 Jahre im Vergleich zu 8.5 Jahre) zumindest zum Teil erklärt werden.

In der Befragung am Ende der sechsten Klasse wurden schließlich 19.9 Prozent der Schüler bzw. 20.7 Prozent der Schülerinnen des Berliner Längsschnitt Medien als übergewichtig oder adipös eingestuft. In der vergleichbaren Altersgruppe der 11-13-jährigen Kinder des KiGGS waren dies 18.3 Prozent der Schüler respektive 18.9 Prozent der Schülerinnen (Kurth, B. \& Schaffrath, 2007). Die 
deutlichen Verschiebungen zwischen den Kategorien übergewichtig und adipös, die für die Daten des Berliner Längsschnitt Medien in der dritten Klasse gegenüber den Daten des KiGGS (7-10) beobachtet werden konnten, entfallen in der sechsten Klasse (Übergewicht: BLM - Jungen: 12.7\%, KiGGS - Jungen: $11.3 \%$; $B L M$ - Mädchen: $13.4 \%$, KiGGS - Mädchen: $11.6 \%$; Adipositas: BLM - Jungen: $7.2 \%$, KiGGS - Jungen: 7.0\%; BLM - Mädchen: $7.3 \%$, KiGGS - Mädchen: $7.3 \%$ ). Da die Schülerinnen und Schüler des Berliner Längsschnitt Medien in der sechsten Klasse hinsichtlich des mittleren Alters (12;5 Jahre) eher dem Mittelwert des KiGGS (12 Jahre) entsprechen, stützen diese Befunde die Annahme, dass die Verschiebungen der dritten Klasse wirklich auf das höhere Durchschnittsalter bei gleichzeitig mit dem Lebensalter steigender Prävalenz von Adipositas zu erklären sind.

Darüber hinaus konnten die Befunde einer im KiGGS beobachteten Abhängigkeit des Übergewichts bzw. der Adipositas vom Migrationshintergrund sowie vom sozioökonomischen Status der Kinder und Jugendlichen bestätigt werden (vgl. Kurth, B. \& Schaffrath, 2007).

\section{Zu 1.) Betrachtung des Zusammenhangs Mediennutzung und Fettleibig-} keit unter Berücksichtigung von Drittvariablen. Zur Beantwortung der Fragestellung wurden quer- wie längsschnittliche Partialkorrelationen berechnet, in denen für den Einfluss des familiären Wohlstands sowie des Bildungsniveaus im Elternhaus kontrolliert wurde, da diese eng mit beiden zu untersuchenden Variablen verbunden sind. Als Ergebnis zeigte sich, dass hinsichtlich der täglichen Mediennutzungszeiten an einem Schultag lediglich für das Fernsehen geringe, jedoch statistisch bedeutsame, negative Zusammenhänge mit dem BMI der Kinder beobachtet werden konnten. Für die täglichen Mediennutzungszeiten an einem Samstag traf dies vor allem auf höhere Computerspielzeiten zu. Somit stehen diese Befunde in einer Tradition der wenigen Studien, die für Computer- und Fernsehnutzung differenzielle Ergebnisse berichtet haben (vgl. Wake et al., 2003; Janssen et al., 2004; Burke et al., 2006; Mendoza et al., 2007; Aucote \& Cooper, 2009; Bishwalata et al., 2010; del Mar Bibiloni et al., 2010). Interessant ist an den Befunden des Berliner Längsschnitt Medien jedoch, dass einem Medium nicht per se ein Zusammenhang abgesprochen wird, sondern dass dieser Zusammenhang lediglich in Abhängigkeit der betrachteten Medienvariable (Nutzungszeiten an einem Schultag vs. Nutzungszeiten an einem Samstag) auftritt. Wie kann man einen derartigen Befund nun erklären? Eine mögliche Erklärung besteht in evtl. unterschiedlichen wöchentlichen Mediennutzungszeiten für Fernsehen und Computerspielen, insbesondere in der Gruppe der Übergewichtigen, mit relativ höheren Fernsehnutzungszeiten unter der Woche und relativ höheren Computerspielzeiten am Wochenende (in der anderen Richtung haben Normalgewichtige möglicherweise an Schultagen relativ geringere Fernsehnutzungszei- 
ten und am Wochenende relativ geringere Computerspielzeiten). Eine zweite mögliche Erklärung besteht in der Rolle der berücksichtigten Kontrollvariablen. So erbrachten hier nicht berichtete Korrelationsberechnungen ohne Kontrollvariablen für das Fernsehen deutlich mehr statistisch bedeutsame Zusammenhänge am Wochenende und für die Computerspiele deutlich mehr statistisch bedeutsame Zusammenhänge für den Schultag, d. h. durch die Berücksichtigung der beiden Kontrollvariablen Bildungsniveau und Wohlstand hat sich die Bedeutsamkeit der Zusammenhänge verändert. Möglicherweise sind unterschiedliche Mediennutzungsmuster am Wochenende und unter der Woche auch von den beiden Kontrollvariablen abhängig. Zugegebener Maßen sind beide Erklärungen angesichts der insgesamt geringen Zusammenhänge von $r=.10$ bis $r=.17$ und der Tatsache, dass die statistisch nicht bedeutsamen Zusammenhänge meist auch in dieselbe Richtung weisen wohl als spekulativ zu bezeichnen (vielleicht handelt es sich auch nur um ein methodisches Artefakt). Dennoch, eins wird daraus deutlich: die Wichtigkeit der Berücksichtigung von Kontrollvariablen sowie die Betrachtung verschiedener Medien in ihrer Bedeutung für die Entwicklung von Fettleibigkeit.

Mit den Fragen „Wie oft läuft der Fernseher bei euch zu Hause während des Abendbrots?“ bzw. „Wie oft läuft der Fernseher bei euch zu Hause während des Essens?" konnte zudem gezeigt werden, dass Kinder, bei denen der Fernseher während des Essens läuft, unabhängig vom familiären Wohlstand und dem Bildungsniveau in ihrem Elternhaus, im Mittel einen höheren BMI haben und somit das Risiko von Übergewicht oder Adipositas durch dieses Verhalten erhöht bzw. gestärkt wird (vgl. hierzu die Ergebnisse von MacFarlane et al., 2009).

$\mathrm{Zu}$ 2.) Können Befunde hinsichtlich einer Wirkungsvermutung im Gegensatz zu einer Selektion im Längsschnitt bestätigt werden? Erste Anhaltspunkte zur Beantwortung dieser Frage sowie zur differenziellen Bedeutung der einzelnen Mediennutzungsvariablen für die Entwicklung von Übergewicht bzw. Adipositas liefern die berechneten längsschnittlichen Partialkorrelationen. Diese legten vor allem eine Wechselwirkung zwischen beiden Medien und dem BMI der Schülerinnen und Schüler nahe, d. h., unabhängig vom familiären Wohlstand und dem Bildungsniveau im Elternhaus spielten übergewichtige und adipöse Kinder länger Computerspiele und schauten länger Fernsehen, was wiederum das Risiko einer Fettleibigkeit, womöglich aufgrund des Bewegungsmangels, erhöhte. Aufgrund der berechneten Korrelationen zeichnete sich also ab, dass auch bei der Betrachtung des Zusammenhangs von Mediennutzug und Fettleibigkeit weder von einer einseitigen Wirkungsvermutung noch einer Selektion auszugehen ist. 
Die berechneten Strukturgleichungsmodelle ${ }^{214}$ zum längsschnittlichen Zusammenhang von Mediennutzung und Fettleibigkeit konnten diesen Befund jedoch nicht bestätigen. Sie beantworteten die Frage nach Wirkung oder Selektion jedoch wiederum recht eindeutig: Im Gegensatz zu allen anderen untersuchten Bereichen ist für den Zusammenhang zwischen Mediennutzung und Fettleibigkeit von einer Selektion in dem Sinne, dass übergewichtige Kinder auch mehr Medien konsumieren, auszugehen. Dieser Befund ist jedoch vor allem auf die Mädchen zurückzuführen: Wie bereits für den Zusammenhang von Mediennutzungszeiten und Schulnoten berichtet, legt das Modell der Mädchen nahe, dass Mädchen mit einem hohen BMI verstärkt Zeit mit Fernsehprogrammen und Computerspielen verbringen (ohne eine Wirkung in die andere Richtung). Bei insgesamt geringeren (und fast durchwegs nicht statistisch bedeutsamen) Pfadkoeffizienten könnte für die Jungen noch eher eine wechselnde Beeinflussung in zeitlicher Abfolge, vergleichbar mit den Befunden zur Schulleistung, geschlossen werden. Zur Erklärung des Gesamtbefundes einer Selektion dürften sich wohl am ehesten Modelle eignen, die Fettleibigkeit und hohen Medienkonsum in den Gesamtkontext eines „sedentary lifestyle“, mit wenig körperlicher Bewegung einordnen (vgl. Crespo et al., 2001; Storey et al., 2003; Janz et al., 2002; Utter et al., 2006; Laxmaiah et al., 2007; Singh et al., 2008; Xu et al., 2008; de Gouw et al., 2010; Liou et al., 2010; Oliver et al., 2010; Papandreou et al., 2010; Rivera et al., 2010; Lehto et al., 2011; Perez et al., 2011; Tremblay \& Willms, 2003; Stettler et al., 2004; Kautiainen et al., 2005; Wang, Y. et al., 2007; Yen, C. F. et al., 2010; Lagiou \& Parava, 2008; Danielsen et al., 2011). Aber auch die Annahme, dass sich übergewichtige Kinder, da sie eher unbeliebt bzw. unsportlich sind, in die digitalen Welten flüchten, um hier selbstwertdienliche Erfahrungen zu machen, ist naheliegend.

Zusammengefasst zeigten sich zwischen Medienkonsum und Fettleibigkeit im Vergleich der unterschiedlichen Konstrukte, welche im Berliner Längsschnitt Medien untersucht wurden, die geringsten Zusammenhänge. Möglicherweise spielen hier die im internationalen Vergleich niedrigen Mediennutzungszeiten der Berliner Grundschülerinnen und Grundschüler eine besondere Rolle, da in anderen Studien Zusammenhänge oft mit deutlich höheren Mediennutzungszeiten berichtet wurden. Besonders deutlich wird an diesen Befunden, dass die Nut-

214 Aufgrund der Befunde der unterschiedlichen Zusammenhänge von schultäglichen und samstäglichen Computerspiel- und Fernsehzeiten mit Übergewicht und Fettleibigkeit, wurde in den Strukturgleichungsmodellen der wöchentliche Durchschnitt beider Variablen in einer Gesamtzeit verwendet, so dass der Einfluss beider Variablen berücksichtigt wird. Eine liberalere Testung hätte lediglich die Fernsehzeiten unter der Woche sowie die Computerspielzeiten am Samstag eingeschlossen, da hier am ehesten Zusammenhänge zu erwarten gewesen wären. Ein derartiges Vorgehen wurde aber aufgrund der größeren Realitätsferne verworfen. 
zung elektronischer Bildschirmmedien nur ein weiterer Einflussfaktor im Erklärungsmodell von Adipositas oder Übergewicht sein kann. Dennoch ergeben sich auch weitere Fragen, die durch die berichteten Ergebnisse aufgeworfen wurden. Ist es überhaupt möglich in einem Alter der Kinder, in dem es eine große wachstumsbedingte natürliche Varianz gibt, anhand des BMI Zusammenhänge zwischen Fettleibigkeit und Mediennutzung zu bestimmen? Von Interesse wäre hier natürlich wie sich frühe hohe Mediennutzungszeiten, unter Berücksichtigung wichtiger Einflussvariablen, später im Leben der dann Jugendlichen auswirken. Können hier möglicherweise deutlichere Zusammenhänge beobachtet werden? Dem würden allerdings die Befunde von Henderson (2007) gegenüberstehen, die (allerdings nur für weiße Mädchen) die Vorhersagekraft früher Fernsehnutzungszeiten bis zu den Teens beschränkt; in der Adoleszenz trat dieser Effekt nicht mehr auf. Kann man darüber hinaus wirklich von einem linearen Zusammenhang zwischen den Variablen der Mediennutzung und dem BMI der Kinder ausgehen (so wie es in den verwendeten statistischen Verfahren zu Grunde gelegt wird)? So wird beispielsweise dadurch vernachlässigt, dass ein problematisches Mediennutzungsverhalten, wie es zum Beispiel bei computerspielabhängigen Jugendlichen oder jungen Erwachsenen beobachtet werden kann, sowohl mit einer Untergewichtigkeit aufgrund von Mangelernährung als auch einer Übergewichtigkeit aufgrund falscher hochkalorischer Ernährung mit einer hohen Energiedichte einhergehen kann. Für zukünftige Berechnungen wäre hier sicher die Überprüfung einer U-Funktion oder die Betrachtung spezifischer „Risikogruppen“ gewinnbringend. Die Daten des Berliner Längsschnitts Medien liefern hierfür erste Anhaltspunkte, die insbesondere bei einer längerfristigen Begleitung ins Jugendalter, altersgruppenspezifisch überprüft werden könnten. 